Research Article

\title{
Vibration Characteristics Analysis of Composite Laminated Annular/Circular Plate Using High-Order Shear Deformation Theory
}

\author{
Guodong Jin $\mathbb{D}^{1},{ }^{1}$ Lei Ma $\mathbb{D}^{1},{ }^{1}$ Hong Zhang $\mathbb{D}^{2},{ }^{2}$ and Qingshan Wang $\mathbb{D}^{3}$ \\ ${ }^{1}$ Luoyang Bearing Research Institute Co., Ltd., Luoyang 471000, China \\ ${ }^{2}$ College of Mechanical and Electrical Engineering, Nanjing University of Aeronautics and Astronautics, Nanjing 210016, China \\ ${ }^{3}$ State Key Laboratory of High Performance Complex Manufacturing, Central South University, Changsha 410083, China
}

Correspondence should be addressed to Hong Zhang; zhanghongyuxin@outlook.com

Received 13 April 2021; Revised 12 May 2021; Accepted 21 July 2021; Published 31 July 2021

Academic Editor: Vasudevan Rajamohan

Copyright (C) 2021 Guodong Jin et al. This is an open access article distributed under the Creative Commons Attribution License, which permits unrestricted use, distribution, and reproduction in any medium, provided the original work is properly cited.

In this paper, the free vibration behaviors of composite laminated annular and circular plates under complex elastic boundary constraints are investigated. Firstly, Reddy's high-order shear deformation theory (HSDT) and Jacobi polynomial method are effectively combined to establish the unified vibration analysis model of composite laminated annular and circular plates. Secondly, the simulation of complex elastic boundary and coupling boundary is realized by using artificial virtual spring technology. Then, the energy equation of the composite laminated plate is established by using Rayleigh-Ritz energy technology. Finally, the free vibration solution equation of the laminated plate is obtained through the Hamilton differential principle. The fast and uniform convergence of this method and the accuracy of the calculated results are verified by numerical examples and the model experimental method. On this basis, the parameterization study is conducted, and the effects of material parameters, geometric parameters, spring stiffness values, and lamination scheme on the vibration characteristics of the annular or circular plate are fully discussed, which can provide a theoretical basis for future research.

\section{Introduction}

The composite laminated annular or circular plates are regarded as the basic components of complex structures in various engineering and industrial application. Therefore, in-depth investigation of the free vibration characteristics of this kind of structure is quite meaningful for the vibration reduction, noise reduction, and impact resistance of the plate. Many scholars have been trying to find new methods or improve the existing methods to further study the vibration mechanical properties of the composite plate structure and have made fruitful research results. Among these research results, the commonly used theories are twodimensional equivalent single-layer plate theory, zig-zag theory, layerwise theory, and three-dimensional elastic theory. The equivalent single-layer plate theory mainly includes classical thin plate theory (CPT), first-order shear deformation theory (FSDT), and high-order shear deformation theory (HSDT). Next, these theories are taken as the main line and briefly introduce the existing research results.

According to the Ritz method and CPT, Afsharmanesh et al. [1] solved the forced vibration problem of laminated circular plates resting on a Winkler-type foundation. Parametric research is mainly focused on the various classical edge constraints and fiber orientation. Amabili et al. [2] investigated the free vibrations of a bolted (or riveted) circular plate having elastic constraints variable by using the Rayleigh-Ritz method. Arshid and Khorshidvand [3] studied the free vibration analysis of saturated porous functionally graded (FG) circular plates based on the differential quadrature method (DQM) and CPT. Bahrami and Teimourian [4] presented a wave propagation approach to analyze free vibration of nonuniform annular and circular membranes. As the first theory to be put forward, CPT completely ignores the shear deformation of the plate, so it is 
only suitable for studying the low frequency vibration behavior of thin plates or shells.

Compared with CPT, the FSDT simply considers the effect of shear deformation on structural vibration by introducing the shear factor. Therefore, this theory can easily solve the vibration behaviors of the plate or shell with medium thickness. Mercan et al. [5] investigated important parametric results for annular plates and conical panels with isotropic, laminated, and FG materials by using FSDT and discrete singular convolution (DSC) method. On the basis of FSDT, Viswanathan et al. [6] expanded the Bickley-type spline function approximation to study the vibration problem of the symmetric angle-ply laminated annular and circular plate of variable thickness. Powmya and Narasimhan [7] discussed the free vibration analysis of orthotropic laminated circular and annular plates based on FSDT. They found that the transverse shear effects of orthotropic laminates are more prominent than that of isotropic laminates. Draiche et al. [8] presented an analytical model to predict the static analysis of laminated reinforced composite plates subjected to sinusoidal and uniform loads by using a simple first-order shear deformation theory. Although the FSDT further considers the effect of transverse shear deformation by introducing shear factor, this theory cannot reflect the warping deformation of the plate in the thickness direction more comprehensively.

In order to overcome the limitations of the CPT and FSDT, the HSDT has been developed, which can better describe the vibration of the composite laminated plate. Amabili et al. [9] investigated forced nonlinear vibrations around the frequency of the fundamental mode by introducing a geometrically nonlinear damping model based on third-order shear deformation theory. In addition, he and his team have carried out a series of studies on the nonlinear mechanics of composite plate and shell structures, based on the third-order shear deformation theory [10-12]. Bisadi et al. [13] employed an exact closed-form solution for freely vibrating annular thick plates under traditional boundary constraints based on Reddy's HSDT. Asadi and Jam [14] employed Mori-Tanaka method to calculate the buckling property of annular plates reinforced by carbon nanotubes on the basis of HSDT. The effects of CNTs' volume fractions, orientation angles, boundary conditions, and geometric ratio of the plate had been analyzed. Vinyas et al. [15] studied the free vibration behavior of annular and circular magnetoelectro-elastic plates through the combination of finite element method and HSDT. Hosseini-Hashemi et al. [16] proposed an exact analytical solution to discuss the freely vibrating piezoelectric coupled circular/annular thick plate based on Reddy's HSDT. The boundary constraints of the plate are set to the combinations of free, simply support, or fixed support. Besides, he and his team [17] presented exact closed-form solutions to investigate free vibration behavior of circular thin, moderately thick, and thick plates with classical boundary constraints based on Reddy's HSDT. Compared with the FSDT, the results obtained by HSDT get closer to the results obtained by 3D elastic theory. Najafizadeh and Heydari [18] considered mechanical buckling of FG circular plate with uniform radial compression based on
HSDT. The study found that HSDT accurately predicts the behavior of the FG circular plate, whereas the CPT and FSDT overestimate buckling loads. In addition, some scholars have proposed a simplified higher-order shear deformation theory to further study the natural characteristics of composite plate or shell structures [19-23]. Katariya et al. [24, 25] studied the vibration characteristic of composite structures based on the HSDT and experimental test method. It is not difficult to find that there are still limitations in the study of vibration characteristics of composite laminated plate structures by using HSDT. Especially, for complex elastic boundary conditions of the laminated plate, the research results are relatively few.

According to the limitations of the above research results, this paper effectively combines Reddy's HSDT and Jacobi polynomials method to establish the unified analytical model of the laminated annular plate and circular plate. This analysis model can quickly and accurately obtain the free vibration behaviors of laminated annular and circular plates under complex elastic boundary constraints. Through the parametric study of boundary spring stiffness, the range of elastic boundary conditions is determined. On this basis, several groups of different boundary settings are given. Besides, in order to ensure the continuity when the rotation angle is 360 degrees, the stiffness value of the connection spring is clearly given. Through the parametric study, the effects of geometric parameters, material parameters, ply angle, and other important parameters on the vibration behaviors of the plate structures are effectively revealed. These research results can provide a theoretical basis for future research.

\section{Theoretical Formulations}

2.1. Model Description of the Laminated Annular/Circular Plate. The theoretical analysis model can be established because of the correlation between the geometry and elasticity of the annular plate and the circular plate. According to the relationships between geometry and elasticity of the annular plate and circular plate, the theoretical analysis model can be found in Figure 1. First of all, as shown in Figure 1(a), it is not difficult to find that the cylindrical coordinate system of the plate is built on the midplane. In this coordinate system, $r$-axis represents the radius direction, and the corresponding geometric parameters are internal radius $R_{0}$ and external radius $R_{1}$, respectively. $R$ represents the difference between $R_{1}$ and $R_{0}$. In particular, the circular plate is set when the inner radius is zero. $\theta$-axis represents the rotation direction of the laminated plate, which ranges from 0 to $2 \pi$. $Z$-axis donates the thickness direction of the plate, which ranges from 0 to $h$. Then, the overall displacement functions $U, V$, and $W$ of this kind of plate correspond to $r$-axis, $\theta$-axis, and $z$-axis, respectively.

In this paper, simulations of various complex elastic boundary constraints are achieved by applying artificial virtual spring technology. All in all, spring components are placed evenly at the boundaries of the annular or circular plate. Among them, the number and type of spring components should be determined according to the 


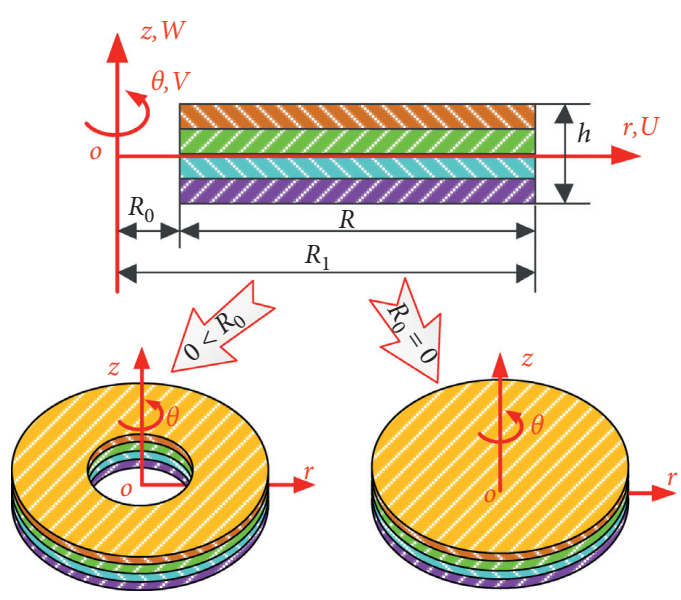

(a)

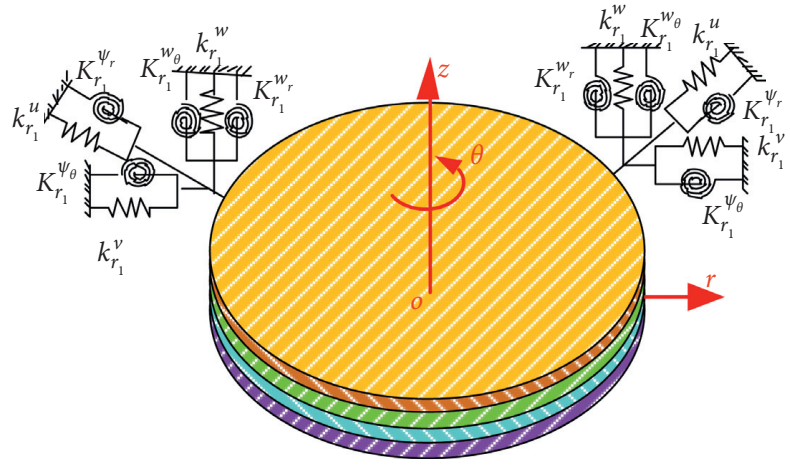

(b)

Figure 1: The unified formulation and boundary conditions for the composite laminated annular plate and circular plate. (a) Coordinate system and unified formulation. (b) Setting of boundary conditions for the circular plate.

displacement function on the middle plane. In this paper, the spring components are shown in Figure 1(b). Taking the outer radius of the laminated circular plate as an example, three linear springs $\left(k_{r_{1}}^{u}, k_{r_{1}}^{v}\right.$, and $\left.k_{r_{1}}^{w}\right)$ and four rotary springs $\left(K_{r_{1}}^{\psi_{r}}, K_{r_{1}}^{\psi_{\theta}}, K_{r_{1}}^{w_{r}}\right.$, and $\left.K_{r_{1}}^{w_{r}}\right)$ form a boundary spring component. When the study model is changed from the annular plate to the circular plate, not only the inner radius is set to 0 but also the stiffness of these boundary springs at this position is set to 0 . Through this series of settings, the simulation of different boundaries can be easily realized.

2.2. Kinematic Relations and Stress-Strain Relations. In the displacement field, the displacements $U$ and $V$ can be extended to the cubic functions of the $z$-axis. Besides, transverse deflection $W$ is assumed to be constant along the thickness direction of the plate. Therefore, the relationship between the global displacement function and the midplane displacement function can be expressed as follows [26, 27]:

$$
\begin{aligned}
U(r, \theta, z, t) & =u(r, \theta, t)+z \psi_{r}(r, \theta, t)+z^{2} \phi_{r}(r, \theta, t)+z^{3} \lambda_{r}(r, \theta, t), \\
V(r, \theta, z, t) & =v(r, \theta, t)+z \psi_{\theta}(r, \theta, t)+z^{2} \phi_{\theta}(r, \theta, t)+z^{3} \lambda_{\theta}(r, \theta, t), \\
W(r, \theta, z, t) & =w(r, \theta, t),
\end{aligned}
$$

where $u, v$, and $w$ are the displacements of the midplane. Then, $\psi_{r}$ and $\psi_{\theta}$ denote the rotation foundations of normal to midplane about the $r$-axis and $\theta$-axis, respectively. Besides, $\phi_{r}, \phi_{\theta}, \lambda_{r}$, and $\lambda_{\theta}$ can be solved by using the vanish of transverse shear stresses at the top and bottom of the plate [26, 27]. Next, a simple solution process will be given.

According to the three-dimensional elasticity theory of the annular/circular plate, the strain-displacement relations in the cylindrical coordinate system can be obtained:

$$
\begin{aligned}
\varepsilon_{r} & =\frac{\partial U}{\partial r}, \\
\varepsilon_{\theta} & =\frac{\partial V}{r \partial \theta}+\frac{U}{r}, \\
\gamma_{r z} & =\frac{\partial W}{\partial r}+\frac{\partial U}{\partial z}, \\
\varepsilon_{r \theta} & =\frac{\partial V}{\partial r}, \\
\varepsilon_{\theta r} & =\frac{\partial U}{r \partial \theta}-\frac{V}{r}, \\
\gamma_{\theta z} & =\frac{\partial W}{r \partial \theta}+\frac{\partial V}{\partial z} .
\end{aligned}
$$

Based on the simple high-order theory put forward by Reddy [26, 27], $\phi_{r}, \phi_{\theta}, \lambda_{r}$, and $\lambda_{\theta}$ can be obtained by setting $\gamma_{r z}(r, \theta, \pm(h / 2))=\gamma_{\theta z}(r, \theta, \pm(h / 2))=0$. So, their expressions can be written as

$$
\begin{aligned}
& \phi_{r}=0, \\
& \lambda_{r}=-\frac{4}{3 h^{2}}\left(\frac{\partial w}{\partial r}+\psi_{r}\right), \\
& \phi_{\theta}=0, \\
& \lambda_{\theta}=-\frac{4}{3 h^{2}}\left(\frac{\partial w}{r \partial \theta}+\psi_{\theta}\right) .
\end{aligned}
$$

In this way, combining equations (1) and (3), the displacement function can be rewritten as follows: 


$$
\begin{aligned}
& U(r, \theta, z, t)=u(r, \theta, t)+g(z) \psi_{r}(r, \theta, t)+f(z)\left[\frac{\partial w(r, \theta, t)}{\partial r}\right], \\
& V(r, \theta, z, t)=v(r, \theta, t)+g(z) \psi_{\theta}(r, \theta, t)+f(z)\left[\frac{\partial w(r, \theta, t)}{r \partial \theta}\right]
\end{aligned}
$$$$
W(r, \theta, z, t)=w(r, \theta, t),
$$

in which $g(z)=z-\left(4 z^{3} / 3 h^{2}\right)$ and $f(z)=-\left(4 z^{3} / 3 h^{2}\right)$. It is not difficult to find from equation (4) that the unknown number of displacement functions involved is the same as that of the FSDT. But the present HSDT explains the parabolic distribution of transverse shear strains along the thickness of the composite laminated annular or circular plate.

Substituting equation (4) into (2), it can be rewritten as

$$
\begin{aligned}
\varepsilon_{r} & =\varepsilon_{r}^{0}+z k_{r}^{0}+z^{2} k_{r}^{1}+z^{3} k_{r}^{2}, \\
\varepsilon_{\theta} & =\varepsilon_{\theta}^{0}+z k_{\theta}^{0}+z^{2} k_{\theta}^{1}+z^{3} k_{\theta}^{2}, \\
\varepsilon_{r \theta} & =\varepsilon_{r \theta}^{0}+z k_{r \theta}^{0}+z^{2} k_{r \theta}^{1}+z^{3} k_{r \theta}^{2}, \\
\varepsilon_{\theta r} & =\varepsilon_{\theta r}^{0}+z k_{\theta r}^{0}+z^{2} k_{\theta r}^{1}+z^{3} k_{\theta r}^{2}, \\
\gamma_{r z} & =\gamma_{r z}^{0}+z k_{r z}^{0}+z^{2} k_{r z}^{1}+z^{3} k_{r z}^{2}, \\
\gamma_{\theta z} & =\gamma_{\theta z}^{0}+z k_{\theta z}^{0}+z^{2} k_{\theta z}^{1}+z^{3} k_{\theta z}^{2},
\end{aligned}
$$

where the strain-displacement relationships on the midplane are given in Table 1.

The unidirectional fiber reinforced layers are the basic components of composite laminated structures. Therefore, the stress-strain relationship of the fiber reinforced layer of the laminated plate is focused. It assumes that the proposed plate has $N$ layers. Then, the relationship of stress-strain for the $k$ th layer can be expressed as

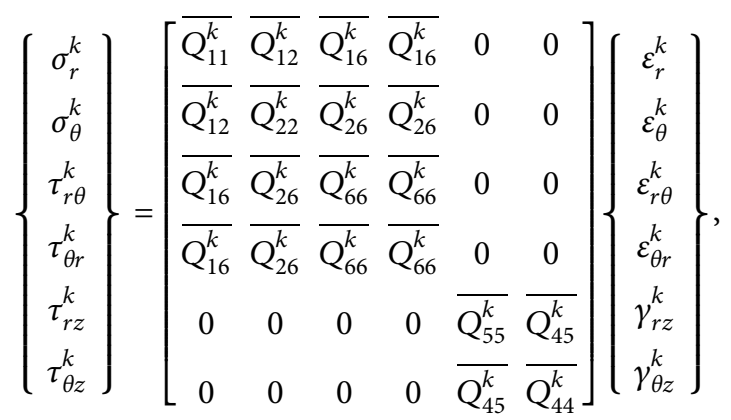

in which $\sigma_{i}^{k}(i=r$ and $\theta)$ and $\tau_{j}^{k}(j=r \theta, \theta r, r z$, and $\theta z)$ denote normal stresses and shear stresses, respectively. Besides, the stiffness coefficients $\overline{Q_{p l}^{k}}$ ( $p$ and $l=1,2,4,5$, and 6) are expressed as

$$
\begin{aligned}
& \overline{Q_{11}^{k}}=Q_{11}^{k} \cos ^{4} \gamma^{k}+2\left(Q_{12}^{k}+2 Q_{66}^{k}\right) \cos ^{2} \gamma^{k} \sin ^{2} \theta^{k}+Q_{22}^{k} \sin ^{4} \gamma^{k}, \\
& \overline{Q_{12}^{k}}=\left(Q_{11}^{k}+Q_{22}^{k}-4 Q_{66}^{k}\right) \cos ^{2} \gamma^{k} \sin ^{2} \gamma^{k}+Q_{12}^{k}\left(\sin ^{4} \gamma^{k}+\cos ^{4} \gamma^{k}\right), \\
& \overline{Q_{22}^{k}}=Q_{11}^{k} \sin ^{4} \gamma^{k}+2\left(Q_{12}^{k}+2 Q_{66}^{k}\right) \cos ^{2} \gamma^{k} \sin ^{2} \gamma^{k}+Q_{22}^{k} \cos ^{4} \gamma^{k}, \\
& \overline{Q_{16}^{k}}=\left(Q_{11}^{k}-Q_{12}^{k}-2 Q_{66}^{k}\right) \cos ^{3} \gamma^{k} \sin \gamma^{k}+\left(Q_{12}^{k}-Q_{22}^{k}+2 Q_{66}^{k}\right) \cos \gamma^{k} \sin ^{3} \gamma^{k}, \\
& \overline{Q_{26}^{k}}=\left(Q_{11}^{k}-Q_{12}^{k}-2 Q_{66}^{k}\right) \cos \gamma^{k} \sin ^{3} \gamma^{k}+\left(Q_{12}^{k}-Q_{22}^{k}+2 Q_{66}^{k}\right) \cos ^{3} \gamma^{k} \sin \gamma^{k}, \\
& \overline{Q_{66}^{k}}=\left(Q_{11}^{k}+Q_{22}^{k}-2 Q_{12}^{k}-2 Q_{66}^{k}\right) \cos ^{2} \gamma^{k} \sin ^{2} \gamma^{k}+Q_{66}^{k}\left(\sin ^{4} \gamma^{k}+\cos ^{4} \gamma^{k}\right), \\
& \overline{Q_{44}^{k}}=Q_{44}^{k} \cos ^{2} \gamma^{k}+Q_{55}^{k} \sin ^{2} \gamma^{k}, \\
& \overline{Q_{45}^{k}}=\left(Q_{55}^{k}-Q_{44}^{k}\right) \cos \gamma^{k} \sin \gamma^{k}, \\
& \overline{Q_{55}^{k}}=Q_{55}^{k} \cos ^{2} \gamma^{k}+Q_{44}^{k} \sin ^{2} \gamma^{k},
\end{aligned}
$$

in which the angle between the fiber direction and the radius direction is defined as $\gamma^{k}$. $Q_{p l}^{k}$ is the material coefficient and is given as 
TABLE 1: The strain-displacement relationships on the mid-plane.

\begin{tabular}{lcccc}
\hline$i$ & $\varepsilon_{i}^{0}\left(\gamma_{i}^{0}\right)$ & $k_{i}^{0}$ & $k_{i}^{1}$ & $k_{i}^{2}$ \\
\hline$r$ & $(\partial u / \partial r)$ & $\left(\partial \psi_{r} / \partial r\right)$ & 0 & $-\left(4 / 3 h^{2}\right)\left(\left(\partial \psi_{r} / \partial r\right)+\left(\partial^{2} w / \partial r^{2}\right)\right)$ \\
$\theta$ & $(\partial v / r \partial \theta)+(u / r)$ & $\left(\partial \psi_{\theta} / r \partial \theta\right)+\left(\psi_{r} / r\right)$ & 0 & $-\left(4 / 3 h^{2}\right)\left(\left(\partial \psi_{\theta} / r \partial \theta\right)+\left(\psi_{r} / r\right)+\left(\partial^{2} w / r^{2} \partial \theta^{2}\right)+(\partial w / r \partial r)\right)$ \\
$r \theta$ & $(\partial v / \partial r)$ & $\left(\partial \psi_{\theta} / \partial r\right)$ & 0 & $-\left(4 / 3 h^{2}\right)\left(\left(\partial \psi_{\theta} / \partial r\right)+\left(\partial^{2} w / r \partial r \partial \theta\right)\right)$ \\
$\theta r$ & $(\partial u / r \partial \theta)-(v / r)$ & $\left(\partial \psi_{r} / r \partial \theta\right)-\left(\psi_{\theta} / r\right)$ & 0 & $-\left(4 / 3 h^{2}\right)\left(\left(\partial \psi_{r} / r \partial \theta\right)+\left(\partial^{2} w / r \partial r \partial \theta\right)-\left(\psi_{\theta} / r\right)-\left(\partial w / r^{2} \partial \theta\right)\right)$ \\
$r z$ & $(\partial w / r)+\psi_{r}$ & 0 & $-\left(4 / h^{2}\right)\left(\psi_{r}+(\partial w / \partial r)\right)$ & 0 \\
$\theta z$ & $(\partial w / r \partial \theta)+\psi_{\theta}$ & 0 & $-\left(4 / h^{2}\right)\left(\psi_{\theta}+(\partial w / r \partial \theta)\right)$ & 0 \\
\hline
\end{tabular}

$$
\begin{aligned}
Q_{11}^{k} & =\frac{E_{1}^{k}}{1-\mu_{12}^{k} \mu_{21}^{k}}, \\
Q_{12}^{k} & =\mu_{21}^{k} Q_{11}^{k}, \\
Q_{22}^{k} & =\frac{E_{2}^{k}}{1-\mu_{12}^{k} \mu_{21}^{k}}, \\
Q_{44}^{k} & =G_{23}^{k}, \\
Q_{55}^{k} & =G_{13}^{k}, \\
Q_{66}^{k} & =G_{12}^{k},
\end{aligned}
$$

where $\mu_{12}^{k}$ and $\mu_{21}^{k}$ are Poisson's ratios. Young's modulus is expressed as $E_{1}^{k}$ and $E_{2}^{k}$. The relationship between Poisson's ratio and Young's modulus is $\mu_{12} E_{2}^{k}=\mu_{21} E_{1}^{k}$. Besides, the shear modulus is denoted as $G_{12}^{k}, G_{13}^{k}$, and $G_{23}^{k}$. When $E_{2}^{k}=$ $E_{1}^{k}$ and $G_{12}^{k}=G_{13}^{k}=G_{23}^{k}=\left(E_{1}^{k} / 2\left(1+\mu_{12}^{k}\right)\right)$, the isotropic plate can be simulated.

So far, the relations between generalized force and strain can be written as the matrix form:

$$
\begin{aligned}
& \left\{\begin{array}{c}
\mathbf{N} \\
M \\
\mathbf{N}^{1} \\
\mathbf{M}^{2}
\end{array}\right\}=\left[\begin{array}{llll}
\mathrm{A} & \mathrm{B} & \mathrm{C} & \mathrm{D} \\
\mathrm{B} & \mathrm{C} & \mathrm{D} & \mathrm{E} \\
\mathrm{C} & \mathrm{D} & \mathrm{E} & \mathrm{F} \\
\mathrm{D} & \mathrm{E} & \mathrm{F} & \mathrm{G}
\end{array}\right]\left\{\begin{array}{c}
\varepsilon \\
k \\
k^{1} \\
k^{2}
\end{array}\right\}, \\
& \left\{\begin{array}{c}
Q \\
P \\
Q^{1} \\
P^{2}
\end{array}\right\}=\left[\begin{array}{cccc}
A_{\kappa} & B_{\kappa} & C_{\kappa} & D_{\kappa} \\
B_{\kappa} & C_{\kappa} & D_{\kappa} & E_{\kappa} \\
C_{\kappa} & D_{\kappa} & E_{\kappa} & F_{\kappa} \\
D_{\kappa} & E_{\kappa} & F_{\kappa} & G_{\kappa}
\end{array}\right]\left\{\begin{array}{c}
\gamma_{z} \\
k_{z} \\
k_{z}^{1} \\
k_{z}^{2}
\end{array}\right\} \\
& \mathbf{N}=\left\{\begin{array}{llll}
N_{r} & N_{\theta} & N_{r \theta} & N_{\theta r}
\end{array}\right\}^{T}, \\
& \mathbf{M}=\left\{\begin{array}{llll}
M_{r} & M_{\theta} & M_{r \theta} & M_{\theta r}
\end{array}\right\}^{T}, \\
& \mathbf{N}^{1}=\left\{\begin{array}{llll}
N_{r}^{1} & N_{\theta}^{1} & N_{r \theta}^{1} & N_{\theta r}^{1}
\end{array}\right\}^{T}, \\
& \mathbf{M}^{2}=\left\{\begin{array}{llll}
M_{r}^{2} & M_{\theta}^{2} & M_{r \theta}^{2} & M_{\theta r}^{2}
\end{array}\right\}^{T}, \\
& \boldsymbol{\varepsilon}=\left\{\begin{array}{llll}
\varepsilon_{r}^{0} & \varepsilon_{\theta}^{0} & \varepsilon_{r \theta}^{0} & \varepsilon_{\theta r}^{0}
\end{array}\right\}^{T},
\end{aligned}
$$

$$
\begin{aligned}
& \mathbf{k}=\left\{\begin{array}{llll}
k_{r}^{0} & k_{\theta}^{0} & k_{r \theta}^{0} & k_{\theta r}^{0}
\end{array}\right\}^{T}, \\
& \mathbf{k}^{1}=\left\{\begin{array}{llll}
k_{r}^{1} & k_{\theta}^{1} & k_{r \theta}^{1} & k_{\theta r}^{1}
\end{array}\right\}^{T}, \\
& \mathbf{k}^{2}=\left\{\begin{array}{llll}
k_{r}^{2} & k_{\theta}^{2} & k_{r \theta}^{2} & k_{\theta r}^{2}
\end{array}\right\}^{T}, \\
& \mathbf{Q}=\left\{\begin{array}{ll}
Q_{r} & Q_{\theta}
\end{array}\right\}^{T}, \\
& \mathbf{Q}=\left\{\begin{array}{ll}
P_{r} & P_{\theta}
\end{array}\right\}^{\mathrm{T}}, \\
& \mathbf{Q}=\left\{\begin{array}{ll}
Q_{r}^{1} & Q_{\theta}^{1}
\end{array}\right\}^{T}, \\
& \mathbf{Q}=\left\{\begin{array}{ll}
P_{r}^{2} & P_{\theta}^{2}
\end{array}\right\}^{T}, \\
& \gamma_{\mathbf{z}}=\left\{\begin{array}{ll}
\gamma_{r z}^{0} & \gamma_{\theta z}^{0}
\end{array}\right\}^{T}, \\
& \mathbf{k}_{\mathbf{z}}=\left\{\begin{array}{ll}
k_{r z}^{0} & k_{\theta z}^{0}
\end{array}\right\}^{T}, \\
& \mathbf{k}_{\mathbf{z}}^{1}= \begin{cases}k_{r z}^{1} & \left.k_{\theta z}^{1}\right\}^{T},\end{cases} \\
& \mathbf{k}_{\mathbf{z}}^{2}=\left\{\begin{array}{ll}
k_{r z}^{2} & k_{\theta z}^{2}
\end{array}\right\}^{T}, \\
& \mathbf{A}=\left[\begin{array}{llll}
A_{11} & A_{12} & A_{16} & A_{16} \\
A_{12} & A_{22} & A_{26} & A_{26} \\
A_{16} & A_{26} & A_{66} & A_{66} \\
A_{16} & A_{26} & A_{66} & A_{66}
\end{array}\right], \\
& \mathbf{B}=\left[\begin{array}{llll}
B_{11} & B_{12} & B_{16} & B_{16} \\
B_{12} & B_{22} & B_{26} & B_{26} \\
B_{16} & B_{26} & B_{66} & B_{66} \\
B_{16} & B_{26} & B_{66} & B_{66}
\end{array}\right], \\
& \mathbf{C}=\left[\begin{array}{llll}
C_{11} & C_{12} & C_{16} & C_{16} \\
C_{12} & C_{22} & C_{26} & C_{26} \\
C_{16} & C_{26} & C_{66} & C_{66} \\
C_{16} & C_{26} & C_{66} & C_{66}
\end{array}\right],
\end{aligned}
$$




$$
\begin{aligned}
& \mathbf{D}=\left[\begin{array}{llll}
D_{11} & D_{12} & D_{16} & D_{16} \\
D_{12} & D_{22} & D_{26} & D_{26} \\
D_{16} & D_{26} & D_{66} & D_{66} \\
D_{16} & D_{26} & D_{66} & D_{66}
\end{array}\right], \\
& \mathbf{G}_{\mathbf{\kappa}}=\left[\begin{array}{ll}
G_{55} & G_{45} \\
G_{45} & G_{44}
\end{array}\right], \\
& \left\{\begin{array}{lllllll}
A_{p l} & B_{p l} & C_{p l} & D_{p l} & E_{p l} & F_{p l} & G_{p l}
\end{array}\right\} \\
& =\sum_{k=1}^{N} \overline{Q_{p l}^{k}} \int_{Z_{k}}^{Z_{k+1}}\left\{\begin{array}{lllllll}
1 & z & z^{2} & z^{3} & z^{4} & z^{5} & z^{6}
\end{array}\right\} \mathrm{d} z \text {, }
\end{aligned}
$$$$
\mathbf{E}=\left[\begin{array}{llll}
E_{11} & E_{12} & E_{16} & E_{16} \\
E_{12} & E_{22} & E_{26} & E_{26} \\
E_{16} & E_{26} & E_{66} & E_{66} \\
E_{16} & E_{26} & E_{66} & E_{66}
\end{array}\right],
$$$$
\mathbf{F}=\left[\begin{array}{llll}
F_{11} & F_{12} & F_{16} & F_{16} \\
F_{12} & F_{22} & F_{26} & F_{26} \\
F_{16} & F_{26} & F_{66} & F_{66} \\
F_{16} & F_{26} & F_{66} & F_{66}
\end{array}\right],
$$$$
\mathbf{G}=\left[\begin{array}{llll}
G_{11} & G_{12} & G_{16} & G_{16} \\
G_{12} & G_{22} & G_{26} & G_{26} \\
G_{16} & G_{26} & G_{66} & G_{66} \\
G_{16} & G_{26} & G_{66} & G_{66}
\end{array}\right] \text {, }
$$$$
\mathbf{A}_{\kappa}=\left[\begin{array}{ll}
A_{55} & A_{45} \\
A_{45} & A_{44}
\end{array}\right] \text {, }
$$$$
\mathbf{B}_{\mathbf{\kappa}}=\left[\begin{array}{ll}
B_{55} & B_{45} \\
B_{45} & B_{44}
\end{array}\right],
$$$$
\mathbf{C}_{\boldsymbol{\kappa}}=\left[\begin{array}{ll}
C_{55} & C_{45} \\
C_{45} & C_{44}
\end{array}\right] \text {, }
$$$$
\mathbf{D}_{\mathbf{\kappa}}=\left[\begin{array}{cc}
D_{55} & D_{45} \\
D_{45} & D_{44}
\end{array}\right] \text {, }
$$$$
\mathbf{E}_{\mathbf{\kappa}}=\left[\begin{array}{ll}
E_{55} & E_{45} \\
E_{45} & E_{44}
\end{array}\right],
$$$$
\mathbf{F}_{\mathbf{\kappa}}=\left[\begin{array}{ll}
F_{55} & F_{45} \\
F_{45} & F_{44}
\end{array}\right] \text {, }
$$

in which $A_{p l}, B_{p l}, C_{p l}, D_{p l}, E_{p l}, F_{p l}$, and $G_{p l}$ are marked as the stiffness coefficients. Besides, $Z_{k}$ is the thickness coordinate value of the under surface of the $k$ th layer plate. $Z_{k+1}$ is the thickness coordinate value of the upper surface of the $k$ th layer plate. So, the thickness of this layer plate is $z_{k}=Z_{k+1}-Z_{k}$.

2.3. Energy Equation of Laminated Annular/Circular Plate. In order to decrease the requirement of displacement function selection, a kind of multisegment segmentation technique is extended which can effectively relax the boundary constraints of the laminated annular or circular plate. Specifically, the laminate structure is subdivided into $N_{r}$ segments along the $r$-axis direction. Then, a penalty parameter is introduced to ensure the continuity and boundary constraints between segments. Firstly, the general expression of the Lagrange energy equation $L$ for composite laminated annular or circular plates is given, which fully considers the effect of boundary constraints:

$$
L=\sum_{q}^{N_{r}}\left(T_{q}-U_{q}\right)-\sum_{q}^{N_{r}-1} V_{\mathrm{cp}}^{q}-V_{\mathrm{BC}},
$$

where the kinetic energy and potential energy of the $q$ th segment plate are expressed by the symbols $T_{q}$ and $U_{q}$. When the plate is segmented, the coupling potential energy is introduced, which is represented by symbol $V_{\mathrm{cp}}^{q}$. For the whole plate, the total kinetic energy and total potential energy should be the superposition of $N_{r}$ segments $T_{q}$ and $U_{q}$, while the total coupling potential energy should be the superposition of $\left(N_{r}-1\right)$ segment $V_{\mathrm{cp}}^{q}$. According to the boundary spring simulation technique, the spring potential stored at the plate boundary can be represented by symbol $V_{\mathrm{BC}}$. Next, according to Reddy's HSDT, their specific expressions are given.

For the $q$ th segment plate, the kinetic energy $T_{q}$ is expressed as 


$$
\begin{aligned}
T_{q}= & \frac{1}{2} \int_{r_{q}}^{r_{q+1}} \int_{0}^{2 \pi}\left\{I_{0}\left[(\dot{u})^{2}+(\dot{v})^{2}+(\dot{w})^{2}\right]+2 I_{1}\left[\dot{u} \dot{\psi}_{r}+\dot{v} \dot{\psi}_{\theta}\right]+I_{2}\left[\left(\dot{\psi}_{r}\right)^{2}+\left(\dot{\psi}_{\theta}\right)^{2}\right]\right. \\
& \left.-\frac{8}{3 h^{2}} I_{3}\left[\dot{u} \frac{\partial \dot{w}}{\partial r}+\dot{u}_{r}+\dot{v} \frac{\partial \dot{w}}{r \partial \theta}+\dot{v} \dot{\psi}_{\theta}\right]-\frac{8}{3 h^{2}} I_{4}\left[\left(\dot{\psi}_{r}\right)^{2}+\left(\dot{\psi}_{\theta}\right)^{2}+\dot{\psi}_{r} \frac{\partial \dot{w}}{\partial r}+\dot{\psi}_{\theta} \frac{\partial \dot{w}}{r \partial \theta}\right]+\frac{16}{9 h^{4}} I_{6}\left[\left(\frac{\partial \dot{w}}{\partial r}+\dot{\psi}_{r}\right)^{2}+\left(\frac{\partial \dot{w}}{r \partial \theta}+\dot{\psi}_{\theta}\right)^{2}\right]\right\} r \mathrm{~d} r \mathrm{~d} \theta,
\end{aligned}
$$

in which

$$
\left\{I_{0}, I_{1}, I_{2}, I_{3}, I_{4}, I_{5}, I_{6}\right\}=\int_{Z_{k}}^{Z_{k+1}} \rho(z)\left\{1, z, z^{2}, z^{3}, z^{4}, z^{5}, z^{6}\right\} \mathrm{d} z .
$$

The specific expression of $U_{q}$ stored in the $q$ th segment laminated plate is

$$
\begin{aligned}
U_{q}= & \frac{1}{2} \int_{r_{q}}^{r_{q+1}} \int_{0}^{2 \pi}\left\{\boldsymbol{\varepsilon}^{T}\left(\mathbf{A} \boldsymbol{\varepsilon}+\mathbf{B k}+\mathbf{C} \mathbf{k}^{1}+\mathbf{D} \mathbf{k}^{2}\right)+\gamma_{\mathbf{z}}^{T}\left(\mathbf{A}_{\mathbf{k}} \gamma_{\mathbf{z}}+\mathbf{B}_{\mathbf{k}} \mathbf{k}_{\mathbf{z}}+\mathbf{C}_{\mathbf{k}} \mathbf{k}_{\mathbf{z}}^{1}+\mathbf{D}_{\mathbf{k}} \mathbf{k}_{\mathbf{z}}^{2}\right)+\mathbf{k}^{T}\left(\mathbf{B} \boldsymbol{\varepsilon}+\mathbf{C k}+\mathbf{D} \mathbf{k}^{1}+\mathbf{E k}^{2}\right)\right. \\
& +\mathbf{k}_{\mathbf{z}}^{T}\left(\mathbf{B}_{\mathbf{k}} \gamma_{\mathbf{z}}+\mathbf{C}_{\mathbf{k}} \mathbf{k}_{\mathbf{z}}+\mathbf{D}_{\mathbf{k}} \mathbf{k}_{\mathbf{z}}^{1}+\mathbf{E}_{\mathbf{k}} \mathbf{k}_{\mathbf{z}}^{2}\right)+\left(\mathbf{k}^{1}\right)^{T}\left(\mathbf{C} \boldsymbol{\varepsilon}+\mathbf{D} \mathbf{k}+\mathbf{E} \mathbf{k}^{1}+\mathbf{F} \mathbf{k}^{2}\right)+\left(\mathbf{k}_{\mathbf{z}}^{1}\right)^{T}\left(\mathbf{C}_{\mathbf{k}} \gamma_{\mathbf{z}}+\mathbf{D}_{\mathbf{k}} \mathbf{k}_{\mathbf{z}}+\mathbf{E}_{\mathbf{k}} \mathbf{k}_{\mathbf{z}}^{1}+\mathbf{F}_{\mathbf{k}} \mathbf{k}_{\mathbf{z}}^{2}\right) \\
& \left.+\left(\mathbf{k}^{2}\right)^{T}\left(\mathbf{D} \boldsymbol{\varepsilon}+\mathbf{E} \mathbf{k}+\mathbf{F} \mathbf{k}^{1}+\mathbf{G} \mathbf{k}^{2}\right)+\left(\mathbf{k}_{\mathbf{z}}^{2}\right)^{T}\left(\mathbf{D}_{\mathbf{k}} \gamma_{\mathbf{z}}+\mathbf{E}_{\mathbf{k}} \mathbf{k}_{\mathbf{z}}+\mathbf{F}_{\mathbf{k}} \mathbf{k}_{\mathbf{z}}^{1}+\mathbf{G}_{\mathbf{k}} \mathbf{k}_{\mathbf{z}}^{2}\right)\right\} r \mathrm{~d} r d \theta .
\end{aligned}
$$

As mentioned above, when the plate structure is segmented along the radius direction, the coupling potential energy between adjacent segments is needed to ensure the continuity of displacements. Similarly, the coupling boundary is simulated by virtual spring technology. In short, the coupling springs $\left(k_{c}^{u}, k_{c}^{v}, k_{c}^{w}, K_{c}^{\psi_{r}}, K_{c}^{\psi_{\theta}}, K_{c}^{w_{r}}\right.$, and $\left.K_{c}^{w_{\theta}}\right)$ are added between adjacent segments. Then, the reasonable spring stiffness is set, which will be further discussed in the following numerical analysis. For the $q$ th segment and $(q+1)$ th segment plate, the coupling potential energy $V_{\mathrm{cp}}^{q}$ can be written as

$$
\begin{aligned}
V_{\mathrm{cp}}^{q}= & \frac{1}{2} \int_{0}^{2 \pi}\left\{k_{c}^{u}\left(u_{q}-u_{q+1}\right)^{2}+k_{c}^{v}\left(v_{q}-v_{q+1}\right)^{2}+k_{c}^{w}\left(w_{q}-w_{q+1}\right)^{2}+K_{c}^{\psi_{r}}\left(\psi_{r, q}-\psi_{r, q+1}\right)^{2}+K_{c}^{\psi_{\theta}}\left(\psi_{\theta, q}-\psi_{\theta, q+1}\right)^{2}\right. \\
& \left.+K_{c}^{w_{r}}\left(\frac{\partial w_{q}}{\partial r}-\frac{\partial w_{q+1}}{\partial r}\right)^{2}+K_{c}^{w_{\theta}}\left(\frac{\partial w_{q}}{r \partial \theta}-\frac{\partial w_{q+1}}{r \partial \theta}\right)^{2}\right\} r \mathrm{~d} \theta .
\end{aligned}
$$

There are a lot of research results on the elastic boundary conditions of plates and shells by using artificial virtual spring technology [28-31]. In Figure 1(b), the setting and types of elastic boundary springs have been given. The expressions of coupling potential energy $V_{\mathrm{BC}}$ of the boundary springs are given directly below:

$$
\begin{aligned}
V_{\mathrm{BC}}= & \frac{1}{2} \int_{0}^{2 \pi}\left\{R_{0}\left(k_{r_{0}}^{u} u^{2}+k_{r_{0}}^{v} v^{2}+k_{r_{0}}^{w} w^{2}+K_{r_{0}}^{\psi_{r}} \psi_{r}^{2}+K_{r_{0}}^{\psi_{\theta}} \psi_{\theta}^{2}+K_{r_{0}}^{w_{r}}\left(\frac{\partial w}{\partial r}\right)^{2}+K_{r_{0}}^{w_{\theta}}\left(\frac{\partial w}{r \partial \theta}\right)^{2}\right)_{r=R_{0}}\right. \\
& \left.+R_{1}\left(k_{r_{1}}^{u} u^{2}+k_{r_{1}}^{v} v^{2}+k_{r_{1}}^{w} w^{2}+K_{r_{1}}^{\psi_{r}} \psi_{r}^{2}+K_{r_{1}}^{\psi_{\theta}} \psi_{\theta}^{2}+K_{r_{1}}^{w_{r}}\left(\frac{\partial w}{\partial r}\right)^{2}+K_{r_{1}}^{w_{\theta}}\left(\frac{\partial w}{r \partial \theta}\right)^{2}\right)_{r=R_{1}}\right\} \mathrm{d} \theta .
\end{aligned}
$$


So far, the energy equation of the elastic laminate structure has been clearly expressed. Next, we will focus on the setting of the displacement function on the reference midsurface and the specific solving process of the natural frequencies and mode shapes.

\subsection{Solution Process of Laminated Annular/Circular Plate.} In the previous section, the global energy equation of the laminated annular or circular plate has been established, so this section will solve it by using the Hamilton differential principle. Firstly, it is necessary to determine the concrete displacement function expression of the middle plane. This paper applies the multisegment segmentation technique to investigate the free vibration behaviors of composite annular or circular plates, which can reduce the requirements of choosing displacement functions. Because compared with directly building the displacement function of the whole structure, the displacement function only needs the low order polynomials for the segment structure. Here, the Jacobi orthogonal polynomials are applied to construct displacement functions [32-36], which can be expressed as

$$
\begin{gathered}
u=\sum_{m=0}^{M} \sum_{n=0}^{N} U_{m n, q} P_{m}^{(\alpha, \beta)}(r)[\cos (n \theta)+\sin (n \theta)] e^{j \omega t}, \\
v=\sum_{m=0}^{M} \sum_{n=0}^{N} V_{m n, q} P_{m}^{(\alpha, \beta)}(r)[\cos (n \theta)+\sin (n \theta)] e^{j \omega t}, \\
w=\sum_{m=0}^{M} \sum_{n=0}^{N} W_{m n, q} P_{m}^{(\alpha, \beta)}(r)[\cos (n \theta)+\sin (n \theta)] e^{j \omega t}, \\
\psi_{r}=\sum_{m=0}^{M} \sum_{n=0}^{N} \psi_{m n, q}^{r} P_{m}^{(\alpha, \beta)}(r)[\cos (n \theta)+\sin (n \theta)] e^{j \omega t}, \\
\psi_{\theta}=\sum_{m=0}^{M} \sum_{n=0}^{N} \psi_{m n, q}^{\theta} P_{m}^{(\alpha, \beta)}(r)[\cos (n \theta)+\sin (n \theta)] e^{j \omega t},
\end{gathered}
$$

where the Jacobi coefficients are expressed as $U_{m n, q}, V_{m n, q}$, $W_{m n, q}, \psi_{m n, q}^{r}$, and $\psi_{m n, q}^{\theta}$ for the $q$ th segment plate. The Jacobi polynomial of the $m$ th in the radius direction is set to $P_{m}^{(\alpha, \beta)}$, and $\alpha$ and $\beta$ denote the type of polynomials. Jacobi polynomials are orthogonal polynomials, so we can get Legendre polynomials, Chebyshev polynomials, and so on by setting the values of $\alpha$ and $\beta$. For example, if the values of $\alpha$ and $\beta$ are 0 , the Legendre polynomial will be obtained. When $\alpha=\beta=-0.5$, it is the first kind of Chebyshev polynomial. When $\alpha=\beta=0.5$, it is the second kind of Chebyshev polynomial. This also shows the universality of the Jacobi polynomials. In addition, $n$ represents the number of half wave in the rotation direction. Then, $j, \omega$, and $t$ are pure imaginary number, circular frequency, and time variable.

According to the Hamilton differential principle, the partial derivative of $L$ to the unknown Jacobi coefficient matrix $\mathbf{E}$ is zero, which can be written as

$$
\begin{aligned}
\frac{\partial L}{\partial \mathbf{E}} & =\sum_{q}^{N_{r}}\left(\frac{\partial T_{q}}{\partial \mathbf{E}}-\frac{\partial U_{q}}{\partial \mathbf{E}}\right)-\sum_{q}^{N_{r}-1} \frac{\partial V_{\mathrm{cp}}^{q}}{\partial \mathbf{E}}-\frac{\partial V_{\mathrm{BC}}}{\partial \mathbf{E}}=0, \\
\mathbf{E} & =\left[U_{m n, q} V_{m n, q} W_{m n, q} \psi_{m n, q}^{r} \psi_{m n, q}^{\theta}\right]^{T} .
\end{aligned}
$$

By introducing equations (11)-(15) into (21), a vibration characteristic solution equation can be obtained:

$$
\left(\mathbf{K}+\mathbf{K}_{\mathrm{cp}}+\mathbf{K}_{\mathrm{BC}}-\omega^{2} \mathbf{M}\right) \mathbf{E}=0,
$$

in which $\mathbf{K}, \mathbf{K}_{\mathrm{cp}}$, and $\mathbf{K}_{\mathrm{BC}}$ are stiffness matrix, coupling matrix between segments, and boundary spring matrix, separately. $\mathbf{M}$ denotes the mass matrix. From this equation, it is easy to find that the circular frequencies can be obtained by finding the generalized eigenvector. In addition, the corresponding generalized eigenvectors are introduced in equations (19)-(23), and the modal shapes of the laminated plate could be easily gained.

\section{Numerical Analysis and Discussion}

By effectively combining Reddy's HSDT and Jacobi polynomials, the equations for solving vibration behaviors of laminated annular or circular plates are established. Next, some numerical examples are applied to study the free vibration characteristics of the laminated plate structures. This section mainly studies the convergence and accuracy, new results, and experimental tests. In addition, it should be pointed out that different boundary constraints are represented by a combination of corresponding first characters. Specifically, since there is only one boundary in the direction of the outer radius for a circular plate, there is only one character to represent it. Among them, the character sign corresponding to the fixed support is C, the sign corresponding to the simple support is $\mathrm{S}$, the sign corresponding to the free boundary is $\mathrm{F}$, and the sign corresponding to the elastic support is E. For annular plates with fixed support in the inner radius direction and simply supported in the outer radius direction, the boundary constraint can be expressed as C-S.

3.1. Model Validation. Convergence and accuracy analysis are important indexes for evaluating an algorithm. Therefore, this section focuses on the convergence of this method and the accuracy of the analysis model in the study of vibration characteristics. By parameterizing the stiffness of the boundary spring, different boundary constraints are defined. In addition, the parameterization of the stiffness of the coupling spring between segments is examined. From the expression of the displacement function, it is not difficult to find that the truncated value $M$ of Jacobi polynomial and segment number $N_{r}$ directly represent the convergence of the proposed method. In Figure 2, the convergence of frequency parameters for the laminated annular or circular plate with fixed boundary constraints is investigated. For the laminated plate, the material parameters are set to $E_{1}=20 E_{2}$, $E_{2}=10 \mathrm{GPa}, G_{12}=G_{13}=0.6 E_{2}, G_{23}=0.5 E_{2}, \mu_{12}=0.25$, and $\rho=1600 \mathrm{~kg} / \mathrm{m}^{3}$. The lamination scheme is $\left[0^{\circ} / 90^{\circ}\right]$, and the 
geometric parameters are $R_{1}=1 \mathrm{~m}$ and $h=0.1 \mathrm{~m}$. In addition, the internal radius $R_{0}$ is $0.3 \mathrm{~m}$. Figure 2 shows the curves of the frequency parameters $\Omega=\left(\omega R^{2} / \sqrt{\rho / h^{2} E_{2}}\right)$ of the annular plate and the circular plate with the increase of $M$ or $N_{r}$. It shows intuitively that the frequency parameters of the two types of plate structures converge when $M$ is 8 or $N_{r}$ is 2 . The proposed method shows good and consistent convergence in solving the natural frequency of this structure. In order to ensure the accuracy of the numerical example, the truncated value in the following examples is defined as $M=10$ and $N_{r}=2$.

As previously described, different types of polynomials could be obtained by setting the values of Jacobi parameters $\alpha$ and $\beta$. Table 2 shows error analysis of frequency parameter $\Omega=\omega R_{1} \sqrt{\rho h / A_{11}}$ for the clamped composite laminated annular plate with various Jacobi parameters $\alpha$ and $\beta$. The material parameters are as follows: $E_{1}=15 E_{2}, E_{2}=10 \mathrm{GPa}$, $G_{12}=G_{13}=0.6 E_{2}, G_{23}=0.5 E_{2}, \mu_{12}=0.25$, and $\rho=1500 \mathrm{~kg} /$ $\mathrm{m}^{3}$. The lamination scheme is $\left[0^{\circ} / 90^{\circ} 0^{\circ} / 90^{\circ}\right]$, and the geometric parameters are $R_{0}=1 \mathrm{~m}, R_{1}=3 \mathrm{~m}$, and $h_{p}=0.1 \mathrm{~m}$. It is not difficult to find out from Table 2 that the Jacobi parameters $\alpha$ and $\beta$ have little effect on the calculation results of the frequency parameter $\Omega$ under the accuracy of five effective digits. In addition, the results obtained in this paper are in excellent agreement with those in [37]. If there is no special description, the Jacobi parameters are chosen as $\alpha=\beta=0$ in other calculation examples in this paper.

As mentioned above, the complex boundary conditions are simulated by artificial virtual spring technology. Next, Figure 3 displays the influence of boundary spring values on natural frequency of the laminated annular plate and a circular plate. Through numerical analysis, the variation range of spring stiffness for the elastic boundary can be determined. Except for the boundary constraints, the structural and material parameters of Figure 3 are exactly the same as those used in Table 2. The range of values of the linear boundary spring (defined as $k$ ) and rotary boundary spring (defined as $K$ ) is both $10^{-4}$ to $10^{16}$. It can be seen from Figure 3 that the waterfall plots of the frequencies to the change of boundary spring stiffness values have the same trends. In brief, when the linear boundary spring stiffness value $(k)$ and the rotary boundary spring stiffness value $(K)$ are less than $10^{4}$, the frequency value is 0 corresponding to the free boundary condition. When $k$ and $K$ are higher than $10^{10}$, the frequency reaches the peak corresponding to the clamped boundary condition. Besides, the elastic boundary conditions can be determined when $k$ and $K$ appear in the following four combinations: $10^{4}<k<10^{10}, \quad K<10^{4}$; $10^{4}<k<10^{10}, K>10^{10} ; 10^{4}<K<10^{10}, k<10^{4} ; 10^{4}<K<10^{10}$, $k>10^{10}$. In light of the conclusions above, the values $k$ and $K$ corresponding to different boundary conditions are given in Table 3.

Now, let us focus on the connective spring. Figure 4 displays the influence of the connective spring stiffness value on the natural frequency of laminated annular and circular plates. In Figure 4, the annular plate is simply supported and the circular plate is clamped. Other parameters are exactly the same with Figure 3. Stiffness values of the connective spring vary from $10^{-2}$ to $10^{16}$. The results show that the effect of connective spring stiffness on the natural frequencies is the same whether it is an annular plate under the SS boundary constraint or a circular plate under the $\mathrm{C}$ boundary constraint. The frequencies are almost unaffected when the value of the connective spring stiffness is less than $10^{4}$. Thereafter, the frequencies increase with the increase of the connective spring stiffness until the value of connective spring stiffness is greater than $10^{10}$. To ensure continuity, the value of connective spring stiffness is chosen as $10^{10}$.

So far, a numerical analysis model for free vibration behaviors of the composite laminated annular or circular plate has been established. To further validate the correctness of the proposed method, Table 4 gives the comparisons of the frequency parameter $\Omega=\omega R_{1} \sqrt{\rho h / A_{11}}$ for the laminated annular plate between the results obtained by HSDT and existing research [37]. Obviously, these two methods have a great agreement, which proves the correctness of the present vibration analysis model. In addition, it is not difficult to see from this table that the stronger the boundary constraint, the greater the natural frequency of the plate.

Then, Table 5 shows the comparison of the frequency parameter $\Omega=\left(\omega R_{1}^{2} / \sqrt{\rho / h^{2} E_{2}}\right)$ for the isotropic circular plate with different thickness ratio $h / R_{1}$. The parameters of the clamped circular plate are $E_{1}=E_{2}=185 \mathrm{GPa}$, $G_{12}=G_{13}=G_{23}=7.12 \mathrm{GPa}, \mu_{12}=0.3, \rho=1600 \mathrm{~kg} / \mathrm{m}^{3}$, and $R_{1}=1 \mathrm{~m}$. The results of FEM in the table are obtained by ABAQUS software. It is not difficult to find that the results obtained by the present method are in good agreement with those obtained by FEM when $h / R_{1}$ is 0.2 . In addition, the material used throughout this numerical example is actually an isotropic material. Therefore, the accuracy and universality of the present method in solving isotropic plate structures are also verified by this example. From this table, it can be found that the natural frequency of the plate decreases with the increase of the ratio of $h / R_{1}$.

3.2. Study on Free Vibration Characteristics. In Section 3.1, the vibration analysis model for the composite annular and circular plate based on Reddy's HSDT and Jacobi polynomials method has been established. The correctness and universality of the present method have been validated, too. In this part, we will give some new results and conclusions.

Firstly, Table 6 shows the frequency parameter $\Omega=\omega R_{1} \sqrt{\rho h / A_{11}}$ for the composite annular plate with various radius ratios and boundary constraints. The material parameters are $E_{1}=30 E_{2}, E_{2}=10 \mathrm{GPa}, G_{12}=G_{13}=0.6 E_{2}$, $G_{23}=0.5 E_{2}, \mu_{12}=0.27$, and $\rho=1600 \mathrm{~kg} / \mathrm{m}^{3}$. And, the lamination scheme is $\left[60^{\circ} /-30^{\circ} / 60^{\circ}\right]$. The invariant geometric parameters are $R_{1}=1 \mathrm{~m}$ and $h=0.15 \mathrm{~m}$. It can be found from Table 6 that radius ratios and boundary constraints have a profound impact on the frequency parameter. On the one hand, the frequency parameter increases when the boundary spring stiffness value increases. On the other hand, the influence of the radius ratio on the frequency parameter is more complex and does not show monotonicity. Then, Table 7 gives the frequency parameter $\Omega=\omega R_{1} \sqrt{\rho h / A_{11}}$ for the laminated annular plate with various boundary constraints and thicknesses. The material parameters involved in 


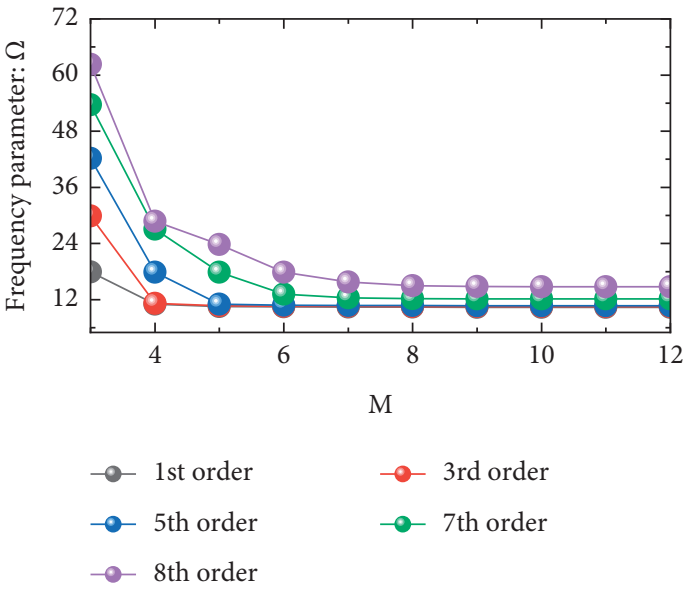

(a)

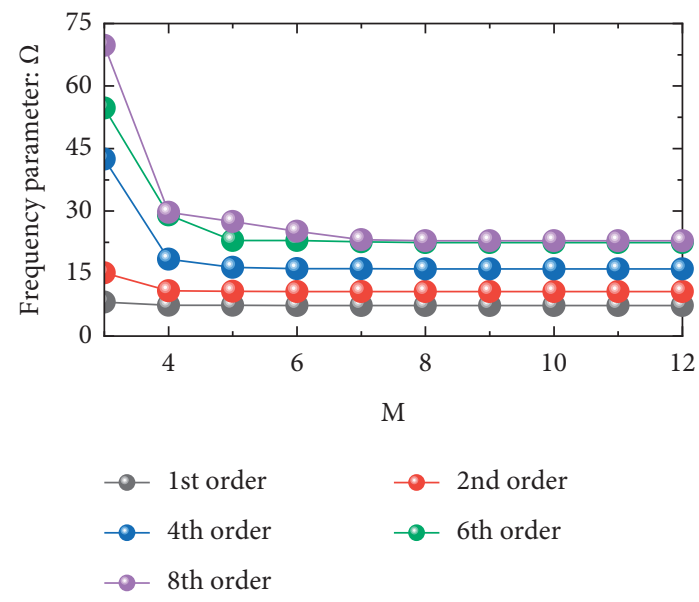

(c)

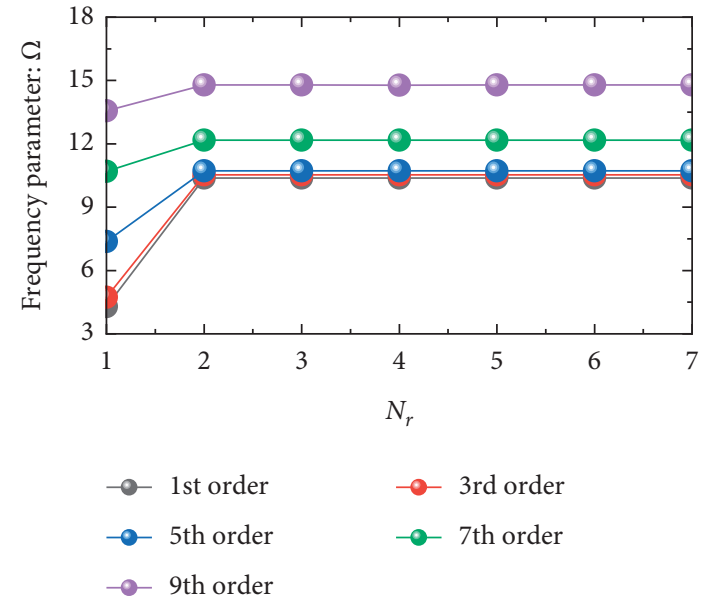

(b)

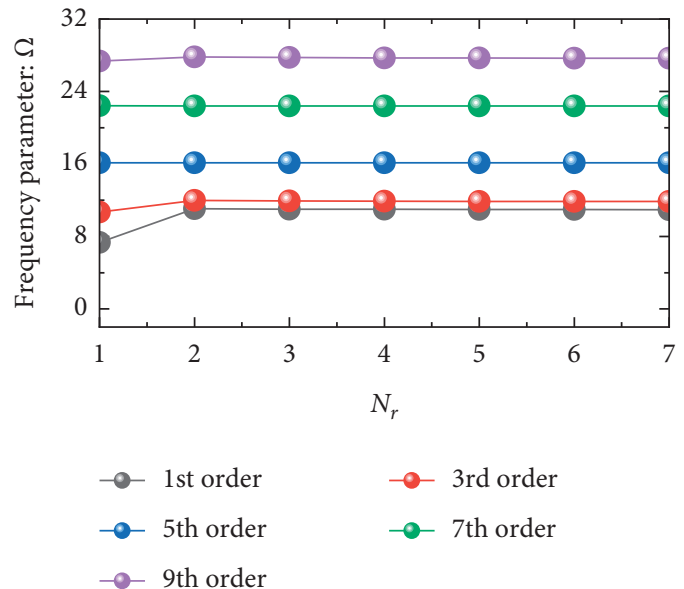

(d)

FIGURE 2: Frequency parameter $\Omega=\left(\omega R^{2} / \sqrt{\rho / h^{2} E_{2}}\right)$ of the composite laminated annular plate and circular plate under fixed boundary constraints with different $\mathrm{M}$ and $N_{r}$. (a) Annular plate under fixed boundary constraints. (b) Circular plate under fixed boundary constraints.

TABLE 2: Error analysis of frequency parameter $\Omega=\omega R_{1} \sqrt{\rho h / A_{11}}$ for Jacobi parameters $\alpha$ and $\beta$ for the annular plate with clamped supported boundary.

\begin{tabular}{|c|c|c|c|c|c|c|}
\hline \multirow{2}{*}{ Method } & \multirow{2}{*}{$(\alpha, \beta)$} & \multicolumn{5}{|c|}{ Mode number } \\
\hline & & 1 & 2 & 3 & 4 & 5 \\
\hline \multirow{10}{*}{ Present } & $(0,0)$ & 0.41618 & 0.41657 & 0.42719 & 0.46641 & 0.54884 \\
\hline & $(0,-0.5)$ & 0.41618 & 0.41657 & 0.42719 & 0.46641 & 0.54884 \\
\hline & $(0,0.5)$ & 0.41618 & 0.41657 & 0.42719 & 0.46641 & 0.54884 \\
\hline & $(-0.5,0)$ & 0.41618 & 0.41657 & 0.42719 & 0.46641 & 0.54884 \\
\hline & $(-0.5,-0.5)$ & 0.41618 & 0.41657 & 0.42719 & 0.46641 & 0.54884 \\
\hline & $(-0.5,0.5)$ & 0.41618 & 0.41657 & 0.42719 & 0.46641 & 0.54884 \\
\hline & $(0.5,0)$ & 0.41618 & 0.41657 & 0.42719 & 0.46641 & 0.54884 \\
\hline & $(0.5,-0.5)$ & 0.41618 & 0.41657 & 0.42719 & 0.46641 & 0.54884 \\
\hline & $(0.5,0.5)$ & 0.41618 & 0.41657 & 0.42719 & 0.46641 & 0.54884 \\
\hline & $(1,1)$ & 0.41618 & 0.41657 & 0.42719 & 0.46641 & 0.54884 \\
\hline Reference [37] & - & 0.41622 & 0.41669 & 0.42751 & 0.46703 & 0.54960 \\
\hline
\end{tabular}

Table 7 are $E_{1}=15 E_{2}, \quad E_{2}=10 \mathrm{GPa}, \quad G_{12}=G_{13}=0.5 E_{2}$, $G_{23}=0.2 E_{2}, \mu_{12}=0.25$, and $\rho=1500 \mathrm{~kg} / \mathrm{m}^{3}$. The lamination scheme is $\left[30^{\circ} /-30^{\circ}\right]$, and $R_{1}=1 \mathrm{~m}$. It is noticeable that the frequency parameter increases when the boundary spring stiffness and plate thickness increase.
Next, Table 8 gives the frequency parameter $\Omega=\left(\omega R_{1}^{2} / \sqrt{\rho / h^{2} E_{2}}\right)$ for the laminated annular plate and a circular plate with diverse anisotropy degrees and lamination schemes. The material parameters implicated in Table 8 are $E_{2}=10 \mathrm{GPa}, G_{12}=G_{13}=0.5 E_{2}, G_{23}=0.6 E_{2}$, 


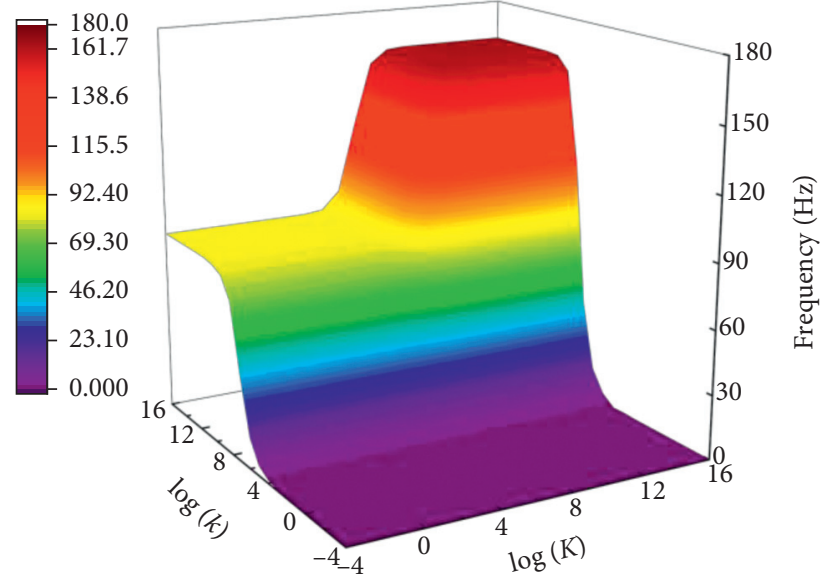

(a)

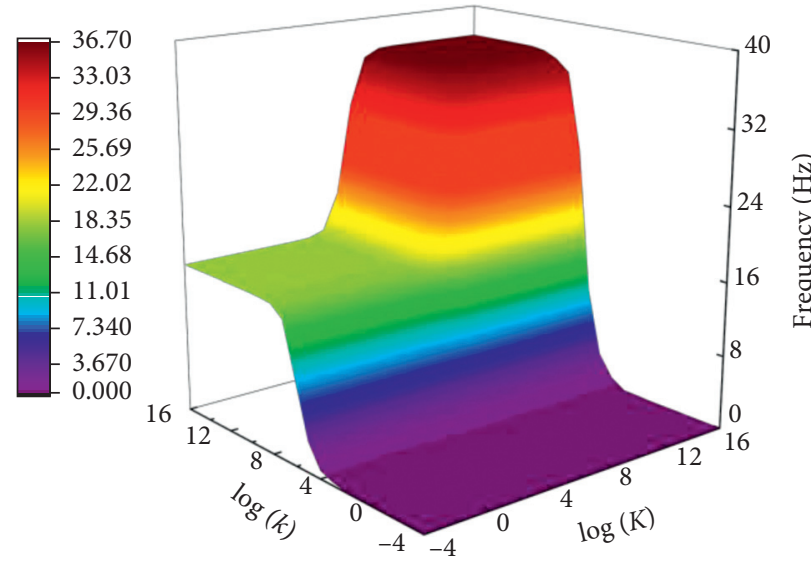

(c)

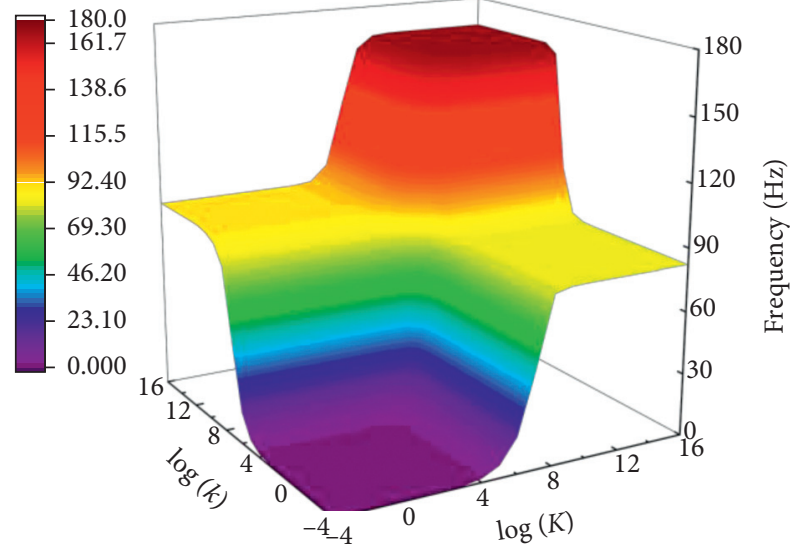

(b)
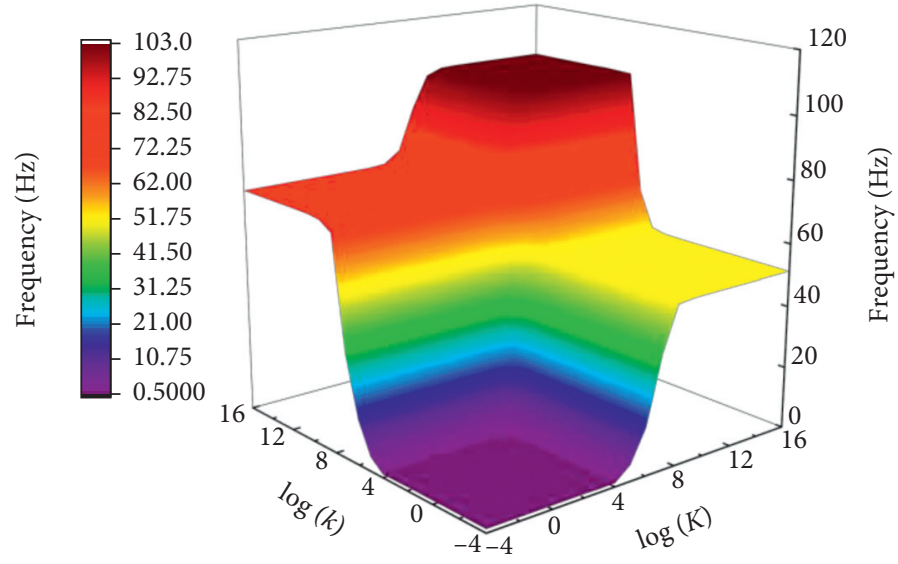

(d)

Figure 3: Effect of the boundary spring value on frequency $(\mathrm{Hz})$ of the composite laminated annular plate and circular plate. (a) Annual plate. (b) Circular plate.

TABLE 3: Stiffness values of the boundary spring corresponding to different boundary conditions.

\begin{tabular}{lccccccc}
\hline BC & \multicolumn{7}{c}{ Stiffness values } \\
& $k_{r_{0}}^{u}$ & $k_{r_{0}}^{v}$ & $k_{r_{0}}^{w}$ & $K_{r_{0}}^{\psi_{r}}$ & $K_{r_{0}}^{\psi_{\theta}}$ & $K_{r_{0}}^{w_{r}}$ & $K_{r_{0}}^{w_{\theta}}$ \\
\hline $\mathrm{F}$ & 0 & 0 & 0 & 0 & 0 & 0 & 0 \\
$\mathrm{C}$ & $10^{14}$ & $10^{14}$ & $10^{14}$ & $10^{14}$ & $10^{14}$ & $10^{14}$ & $10^{14}$ \\
$\mathrm{~S}$ & $10^{14}$ & $10^{14}$ & $10^{14}$ & 0 & 0 & 0 & 0 \\
$\mathrm{E}^{1}$ & $10^{8}$ & $10^{8}$ & $10^{8}$ & $10^{14}$ & $10^{14}$ & $10^{14}$ & $10^{14}$ \\
$\mathrm{E}^{2}$ & $10^{8}$ & $10^{8}$ & $10^{8}$ & $10^{8}$ & $10^{8}$ & $10^{8}$ & $10^{8}$ \\
\hline
\end{tabular}

$\mu_{12}=0.25$, and $\rho=1500 \mathrm{~kg} / \mathrm{m}^{3}$. The geometrical parameters are $R_{1}=1 \mathrm{~m}, h=0.08 \mathrm{~m}$, and $R_{0}=0.3 \mathrm{~m}$ (for the annular plate). The boundary constraints of the annular plate and circular plate are E2-E2 and E2, respectively. It is not difficult to find that the frequency parameter increases with the increase of anisotropy degrees. It is noteworthy that the lamination scheme has an important influence on the frequency parameters. However, due to data limitations, it is difficult to draw general conclusions.

For the sake of fully revealing the effect of material parameters on vibration behaviors of the laminated annular plate and circular plate, the parametric research is conducted. Figure 5 studies the effect of the layer number on natural frequencies of the laminated annular plate and circular plate with fixed boundary constraints. The material parameters are $E_{1}=5 E_{2}, E_{2}=10 \mathrm{GPa}, G_{12}=G_{13}=0.5 E_{2}$, $G_{23}=0.6 E_{2}, \mu_{12}=0.25$, and $\rho=1500 \mathrm{~kg} / \mathrm{m}^{3}$. The geometrical parameters of the clamped plates are $R_{1}=1 \mathrm{~m}, h=0.08 \mathrm{~m}$, and $R_{0}=0.3 \mathrm{~m}$ (for an annular plate). It should be noted that $p$ represents the layer number. And, $\left[0 /-30^{\circ}\right]_{1}$ represents $[0 /$ $\left.-30^{\circ}\right]$, while $\left[0 /-30^{\circ}\right]_{2}$ represents $\left[0 /-30^{\circ} / 0 /-30^{\circ}\right]$, and so on. As can be seen from Figure 5, the frequencies increase when the layer number increases and tend to be stable after the layer number exceeds 4 . Moreover, the frequency changes most significantly when the layer number changes from 1 to 2 .

In addition, for the annular plate, its frequency decreases monotonously with the increase of the ply angle, but not for the circular plate. In order to reveal the effect of the ply angle on the vibration behaviors of the laminated annular and circular plate with fixed boundary constraints, Figure 6 shows the variation of frequency with various ply angles. The lamination scheme used in Figure 6 is chosen as $\left[0 / \gamma^{\circ}\right]$, and $\alpha$ ranges from $10^{\circ}$ to $170^{\circ}$. From Figure 6, it is easy to find that the frequency 


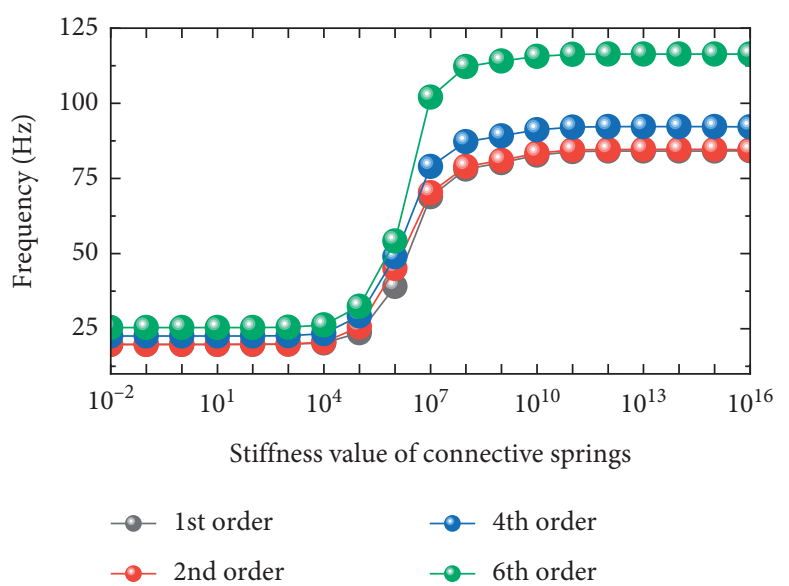

(a)

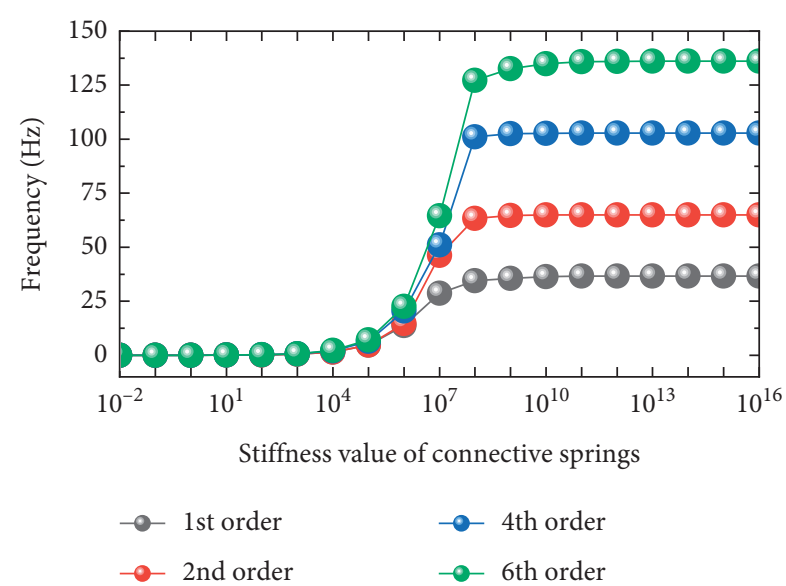

(b)

FIGURE 4: Effect of the connective spring value on frequency $(\mathrm{Hz})$ of the composite laminated annular plate and circular plate. (a) Annular plate under the S-S boundary condition. (b) Circular plate under the C boundary condition.

TABLE 4: Comparison of the frequency parameter $\Omega=\omega R_{1} \sqrt{\rho h / A_{11}}$ for the composite laminated annular plate with different boundary conditions.

\begin{tabular}{|c|c|c|c|c|c|c|}
\hline \multirow{2}{*}{$\mathrm{BC}$} & \multirow{2}{*}{ Method } & \multicolumn{5}{|c|}{ Mode number } \\
\hline & & 1 & 2 & 3 & 4 & 5 \\
\hline \multirow{2}{*}{ F-S } & Present & 0.04245 & 0.08302 & 0.17203 & 0.28055 & 0.37333 \\
\hline & Reference [37] & 0.04255 & 0.08408 & 0.17302 & 0.28132 & 0.37312 \\
\hline \multirow{2}{*}{$\mathrm{F}-\mathrm{C}$} & Present & 0.11687 & 0.14604 & 0.24021 & 0.36739 & 0.49685 \\
\hline & Reference [37] & 0.11671 & 0.14685 & 0.24153 & 0.36835 & 0.49659 \\
\hline \multirow{2}{*}{ S-S } & Present & 0.21671 & 0.21840 & 0.23869 & 0.30135 & 0.40908 \\
\hline & Reference [37] & 0.21669 & 0.21849 & 0.23909 & 0.30212 & 0.40990 \\
\hline \multirow{2}{*}{ S-C } & Present & 0.32832 & 0.32981 & 0.34740 & 0.40547 & 0.51367 \\
\hline & Reference [37] & 0.32825 & 0.32986 & 0.34775 & 0.40619 & 0.51446 \\
\hline \multirow{2}{*}{ C-C } & Present & 0.41618 & 0.41657 & 0.42719 & 0.46641 & 0.54884 \\
\hline & Reference [37] & 0.41622 & 0.41669 & 0.42751 & 0.46703 & 0.54960 \\
\hline
\end{tabular}

TABLE 5: Comparison of the frequency parameter $\Omega=\left(\omega R_{1}^{2} / \sqrt{\rho / h^{2} E_{2}}\right)$ for the composite laminated circular plate with different plate thicknesses.

\begin{tabular}{|c|c|c|c|c|c|c|c|c|}
\hline \multirow{2}{*}{$h / R_{1}$} & \multirow{2}{*}{ Method } & \multicolumn{7}{|c|}{ Mode number } \\
\hline & & 1 & 3 & 5 & 6 & 7 & 9 & 11 \\
\hline \multirow{2}{*}{0.01} & FEM & 3.090 & 6.446 & 10.575 & 12.078 & 15.477 & 18.559 & 21.138 \\
\hline & Present & 3.093 & 6.351 & 10.504 & 12.025 & 15.366 & 18.258 & 20.984 \\
\hline \multirow{2}{*}{0.02} & FEM & 3.087 & 6.435 & 10.548 & 12.043 & 15.420 & 18.481 & 21.034 \\
\hline & Present & 3.091 & 6.338 & 10.476 & 12.001 & 15.309 & 18.176 & 20.882 \\
\hline \multirow{2}{*}{0.05} & FEM & 3.069 & 6.362 & 10.362 & 11.812 & 15.039 & 17.962 & 20.355 \\
\hline & Present & 3.074 & 6.258 & 10.294 & 11.775 & 14.938 & 17.660 & 20.221 \\
\hline \multirow{2}{*}{0.08} & FEM & 3.037 & 6.234 & 10.044 & 11.418 & 14.412 & 17.118 & 19.275 \\
\hline & Present & 3.042 & 6.120 & 9.983 & 11.391 & 14.327 & 16.822 & 19.171 \\
\hline \multirow[b]{2}{*}{0.1} & FEM & 3.008 & 6.123 & 9.778 & 11.092 & 13.906 & 16.445 & 18.435 \\
\hline & Present & 3.013 & 6.001 & 9.724 & 11.073 & 13.835 & 16.155 & 18.355 \\
\hline \multirow{2}{*}{0.15} & FEM & 2.915 & 5.782 & 9.009 & 10.156 & 12.522 & 13.677 & 14.644 \\
\hline & Present & 2.921 & 5.624 & 8.981 & 10.164 & 12.492 & 14.339 & 14.707 \\
\hline \multirow[b]{2}{*}{0.2} & FEM & 2.798 & 5.393 & 8.202 & 9.190 & 10.258 & 11.175 & 11.905 \\
\hline & Present & 2.806 & 5.091 & 8.196 & 9.230 & 11.029 & 11.185 & 11.880 \\
\hline
\end{tabular}

variation is symmetrical with respect to $\left[0 / 90^{\circ}\right]$. On the contrary, when $\alpha$ is less than $90^{\circ}$, the frequency of the annular plate decreases monotonously with the increase of $\gamma$. For the circular plate, the frequency is not significantly affected by the change of the ply angle and does not have monotonicity. 
TABLE 6: The frequency parameter $\Omega=\omega R_{1} \sqrt{\rho h / A_{11}}$ for the composite laminated annular plate with different radius ratios and boundary conditions.

\begin{tabular}{|c|c|c|c|c|c|c|c|c|c|}
\hline \multirow{2}{*}{$R_{0} / R_{1}$} & \multirow{2}{*}{$\mathrm{BC}$} & \multicolumn{8}{|c|}{ Mode number } \\
\hline & & 1 & 2 & 3 & 4 & 5 & 6 & 7 & 8 \\
\hline \multirow{4}{*}{0.1} & C-E1 & 0.2406 & 0.3027 & 0.6400 & 0.6400 & 0.7337 & 0.7337 & 0.7560 & 0.8754 \\
\hline & E1-E1 & 0.1581 & 0.1847 & 0.5236 & 0.6400 & 0.6400 & 0.7013 & 0.7013 & 0.8754 \\
\hline & E1-E2 & 0.1577 & 0.1847 & 0.2920 & 0.2920 & 0.5134 & 0.5190 & 0.5190 & 0.7013 \\
\hline & E2-E2 & 0.1575 & 0.1847 & 0.2748 & 0.2748 & 0.5121 & 0.5185 & 0.5185 & 0.7013 \\
\hline \multirow{4}{*}{0.3} & C-E1 & 0.3222 & 0.8324 & 0.8480 & 0.8480 & 0.9890 & 0.9890 & 1.0692 & 1.1834 \\
\hline & E1-E1 & 0.1725 & 0.1903 & 0.6501 & 0.7515 & 0.7515 & 0.8480 & 0.8480 & 0.9890 \\
\hline & E1-E2 & 0.1722 & 0.1903 & 0.3762 & 0.3762 & 0.5594 & 0.5594 & 0.6332 & 0.7515 \\
\hline & E2-E2 & 0.1720 & 0.1903 & 0.2940 & 0.2940 & 0.5254 & 0.5254 & 0.6183 & 0.7515 \\
\hline \multirow{4}{*}{0.5} & C-E1 & 0.4915 & 1.2811 & 1.2811 & 1.3576 & 1.3576 & 1.4790 & 1.4790 & 1.6372 \\
\hline & E1-E1 & 0.1973 & 0.2074 & 0.7385 & 0.7385 & 0.9624 & 1.2161 & 1.2161 & 1.2811 \\
\hline & E1-E2 & 0.1972 & 0.2074 & 0.5410 & 0.5410 & 0.6993 & 0.6993 & 0.7385 & 0.7385 \\
\hline & E2-E2 & 0.1970 & 0.2074 & 0.3233 & 0.3233 & 0.5601 & 0.5601 & 0.7385 & 0.7385 \\
\hline \multirow{4}{*}{0.8} & C-E1 & 0.4915 & 1.2811 & 1.2811 & 1.3576 & 1.3576 & 1.4790 & 1.4790 & 1.6372 \\
\hline & E1-E1 & 0.2990 & 0.3013 & 0.6889 & 0.6889 & 1.0358 & 1.0358 & 1.1316 & 1.3101 \\
\hline & E1-E2 & 0.2990 & 0.3013 & 0.6889 & 0.6889 & 1.0358 & 1.0358 & 1.1316 & 1.3101 \\
\hline & E2-E2 & 0.2989 & 0.3013 & 0.4561 & 0.4561 & 0.6889 & 0.6889 & 0.7547 & 0.7547 \\
\hline
\end{tabular}

TABLE 7: The frequency parameter $\Omega=\omega R_{1} \sqrt{\rho h / A_{11}}$ for the composite laminated annular plate with different boundary conditions and thicknesses.

\begin{tabular}{llllllllll}
\hline \multirow{2}{*}{ BC } & Mode number & & & \multicolumn{3}{c}{$H$} & & \\
& & $0.01 \mathrm{~m}$ & $0.02 \mathrm{~m}$ & $0.04 \mathrm{~m}$ & $0.08 \mathrm{~m}$ & $0.10 \mathrm{~m}$ & $0.14 \mathrm{~m}$ & $0.18 \mathrm{~m}$ & $0.20 \mathrm{~m}$ \\
\hline \multirow{3}{S}{} & 1 & 0.0064 & 0.0127 & 0.0253 & 0.0504 & 0.0628 & 0.0871 & 0.1107 & 0.1221 \\
& 3 & 0.0286 & 0.0566 & 0.1093 & 0.2271 & 0.2681 & 0.3424 & 0.4010 & 0.4251 \\
& 5 & 0.0464 & 0.0919 & 0.1799 & 0.3368 & 0.4030 & 0.5109 & 0.5904 & 0.6216 \\
\hline & 1 & 0.0175 & 0.0347 & 0.0675 & 0.1122 & 0.1187 & 0.1147 & 0.1057 & 0.1014 \\
E1 & 3 & 0.0411 & 0.0805 & 0.1536 & 0.1837 & 0.1646 & 0.1394 & 0.1231 & 0.1168 \\
& 5 & 0.0615 & 0.1212 & 0.2350 & 0.2669 & 0.2834 & 0.3376 & 0.3967 & 0.4245 \\
\hline & 1 & 0.0176 & 0.0347 & 0.0673 & 0.1106 & 0.1166 & 0.1124 & 0.1037 & 0.0995 \\
E2 & 3 & 0.0409 & 0.0801 & 0.1476 & 0.1834 & 0.1642 & 0.1389 & 0.1226 & 0.1163 \\
& 5 & 0.0617 & 0.1208 & 0.2287 & 0.2335 & 0.2320 & 0.2223 & 0.2141 & 0.2108 \\
\hline & 1 & 0.0175 & 0.0348 & 0.0690 & 0.1343 & 0.1645 & 0.2195 & 0.2666 & 0.2873 \\
C & 3 & 0.0415 & 0.0823 & 0.1577 & 0.3194 & 0.3709 & 0.4589 & 0.5234 & 0.5488 \\
& 5 & 0.0614 & 0.1209 & 0.2345 & 0.4259 & 0.5009 & 0.6159 & 0.6958 & 0.7262 \\
\hline
\end{tabular}

TABLE 8: The frequency parameter $\Omega=\left(\omega R_{1}^{2} / \sqrt{\rho / h^{2} E_{2}}\right)$ for the composite laminated annular/circular plate with different anisotropy degrees and lamination schemes.

\begin{tabular}{|c|c|c|c|c|c|c|c|c|c|c|}
\hline \multirow{2}{*}{ Plate type } & \multirow{2}{*}{$E_{1} / E_{2}$} & \multicolumn{3}{|c|}{$\left[0 / 90^{\circ}\right]$} & \multicolumn{3}{|c|}{$[0 / 90 \%]$} & \multicolumn{3}{|c|}{$\left[0 / 90^{\circ} / 0 / 90^{\circ}\right]$} \\
\hline & & 1 & 3 & 5 & 1 & 3 & 5 & 1 & 3 & 5 \\
\hline \multirow{7}{*}{ Annular } & 5 & 3.7759 & 4.0629 & 5.0452 & 3.9101 & 4.0682 & 5.4108 & 3.8286 & 4.0643 & 5.1982 \\
\hline & 10 & 3.8536 & 4.0754 & 5.2271 & 3.9698 & 4.0798 & 5.5790 & 3.9102 & 4.0775 & 5.4256 \\
\hline & 20 & 3.9219 & 4.0847 & 5.4082 & 4.0040 & 4.0881 & 5.6828 & 3.9638 & 4.0876 & 5.6096 \\
\hline & 30 & 3.9549 & 4.0894 & 5.5108 & 4.0174 & 4.0922 & 5.7285 & 3.9852 & 4.0925 & 5.7084 \\
\hline & 40 & 3.9749 & 4.0924 & 5.5813 & 4.0251 & 4.0949 & 5.7568 & 3.9973 & 4.0957 & 5.7798 \\
\hline & 50 & 3.9883 & 4.0947 & 5.6349 & 4.0304 & 4.0970 & 5.7779 & 4.0052 & 4.0981 & 5.8379 \\
\hline & 60 & 3.9981 & 4.0965 & 5.6782 & 4.0345 & 4.0986 & 5.7951 & 4.0110 & 4.0999 & 5.8884 \\
\hline \multirow{7}{*}{ Circular } & 5 & 3.7423 & 6.2217 & 6.8697 & 4.2955 & 6.2044 & 8.0643 & 4.0328 & 6.2224 & 7.4192 \\
\hline & 10 & 4.2170 & 6.2083 & 7.4886 & 4.9093 & 6.1970 & 8.9443 & 4.6722 & 6.2121 & 8.3725 \\
\hline & 20 & 4.7202 & 6.2051 & 8.2009 & 5.3431 & 6.1987 & 9.6122 & 5.1949 & 6.2105 & 9.2159 \\
\hline & 30 & 5.0007 & 6.2069 & 8.6403 & 5.5136 & 6.2021 & 9.9506 & 5.4174 & 6.2122 & 9.6415 \\
\hline & 40 & 5.1819 & 6.2094 & 8.9480 & 5.6051 & 6.2050 & 10.1838 & 5.5401 & 6.2142 & 9.9247 \\
\hline & 50 & 5.3087 & 6.2116 & 9.1804 & 5.6627 & 6.2075 & 10.3653 & 5.6182 & 6.2160 & 10.1405 \\
\hline & 60 & 5.4024 & 6.2137 & 9.3646 & 5.7026 & 6.2096 & 10.5153 & 5.6724 & 6.2175 & 10.3174 \\
\hline
\end{tabular}



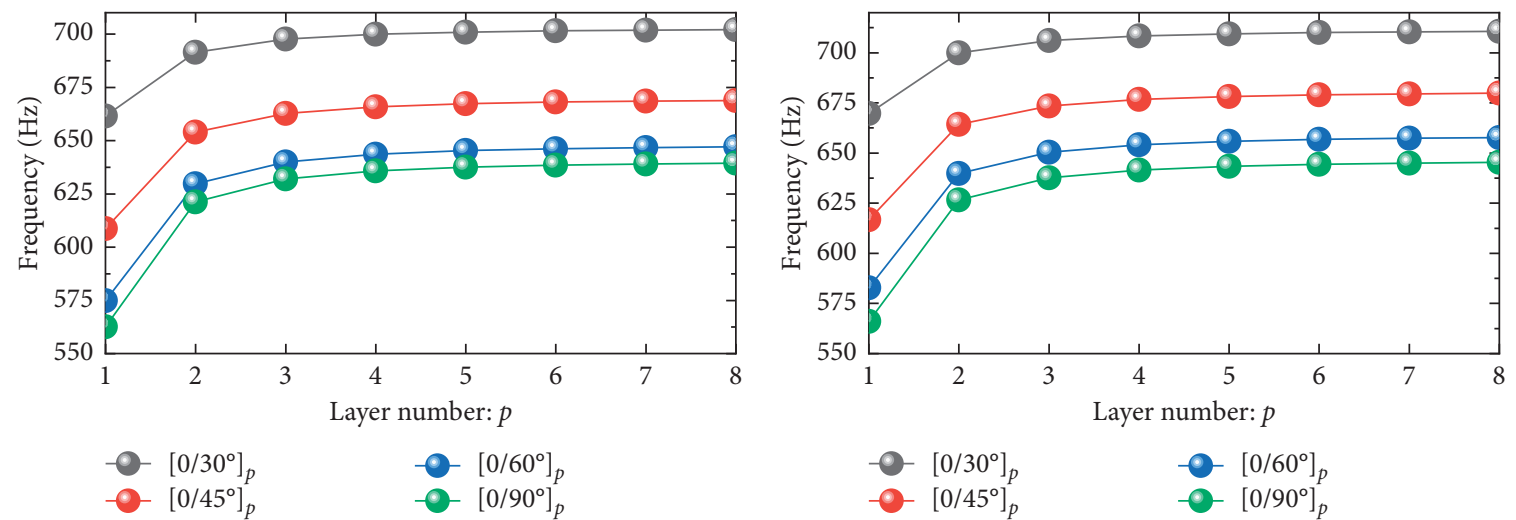

(a)

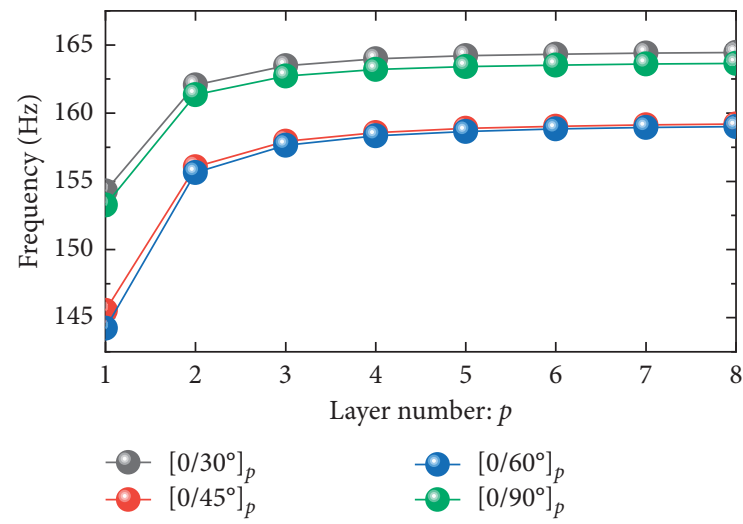

(c)

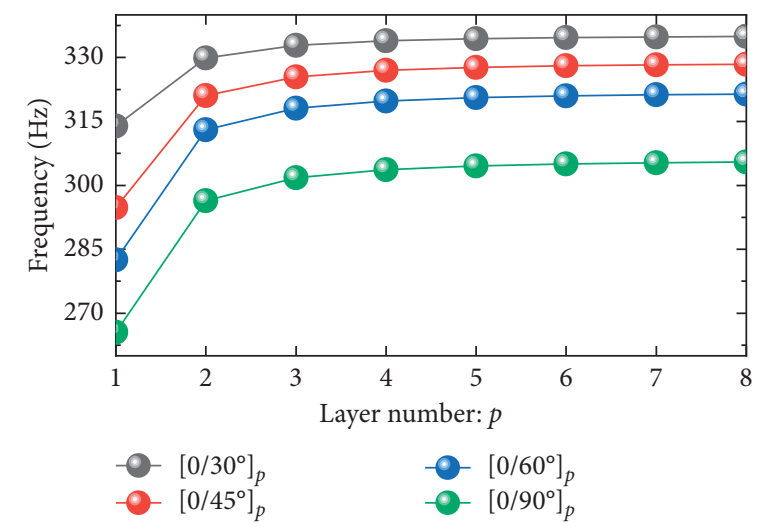

(d)

Figure 5: Effect of the layer number on frequency $(\mathrm{Hz})$ of the fixed supported laminated annular plate and circular plate. (a) Annual plate. (b) Circular plate.

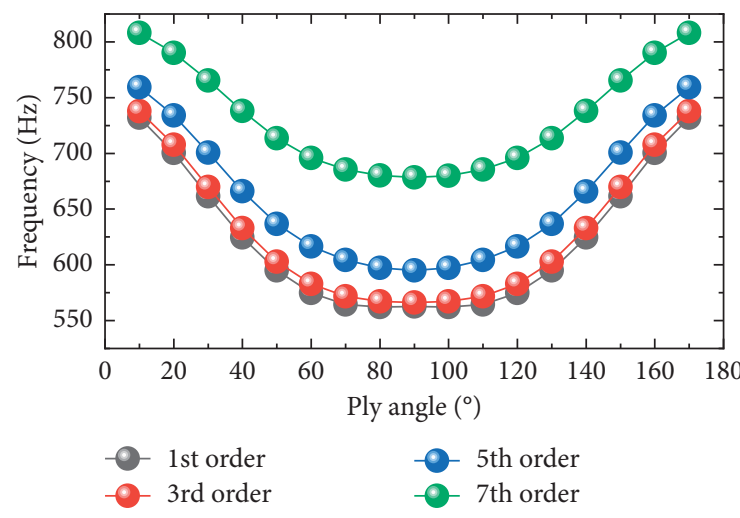

(a)

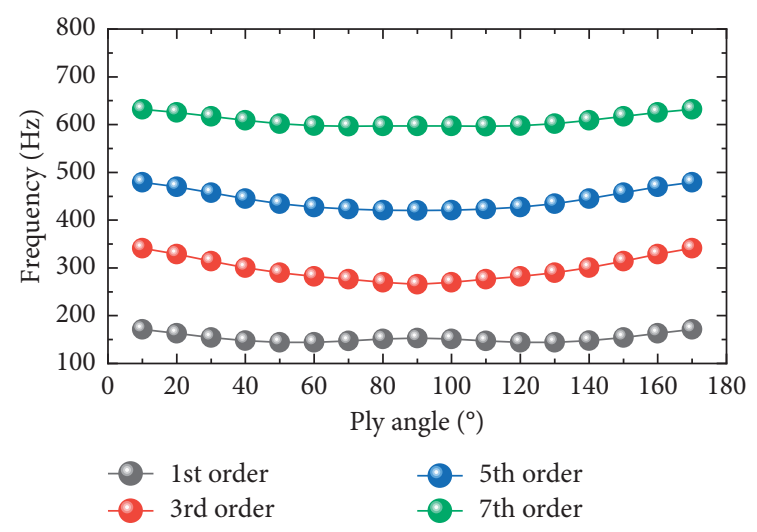

(b)

Figure 6: Effect of the ply angle on frequency $(\mathrm{Hz})$ of the fixed supported laminated annular plate and circular plate. (a) Annular plate. (b) Circular plate.

3.3. Model Experimental Study. In Section 3.2, free vibration behaviors of the laminated annular plate and circular plate are explored in depth based on the high-order shear deformation theory proposed by Reddy and Jacobi polynomials method. However, the previous studies only consider numerical validation. There is a lack of experimental test data to support the qualitative conclusion of the correctness of the above analysis model. In order to more fully verify the correctness of this study, this part applies the model experimental method. At present, equipment and principle of the structural model test can give reliable and more accurate experimental data. In addition, the fixed or free boundary conditions are insensitive to the external environment and easy to get realized. So, the model 
TABLE 9: Experimental instruments for the modal test of rotating plate structures.

\begin{tabular}{|c|c|c|c|}
\hline Name & $\begin{array}{c}\text { Model } \\
\text { number }\end{array}$ & Parameter & Use \\
\hline Hammer & LC-01A & $\begin{array}{l}\text { Hammer head: aluminum hammer head; sensitivity: } 4 \mathrm{pC} / \mathrm{N} \text {; } \\
\text { output signal: electric charge type }\end{array}$ & $\begin{array}{l}\text { Provide incentives for plate } \\
\text { structures }\end{array}$ \\
\hline Charge amplifier & DH5857-1 & $\begin{array}{c}\text { Input resistance: }>10^{11} \Omega \text {; input electric charge: }<10^{5} \mathrm{pC} \text {; } \\
\text { sensitivity: } 0.1,10 \mathrm{mv} / \mathrm{pC}\end{array}$ & $\begin{array}{l}\text { Amplify the excitation force } \\
\text { and acceleration signal }\end{array}$ \\
\hline Acceleration sensor & $1 \mathrm{~A} 116 \mathrm{E}$ & $\begin{array}{l}\text { Weight: } 5.5 \mathrm{~g} \text {; sensitivity: } 10 \mathrm{mv} / \mathrm{m} \cdot \mathrm{s}^{-2} \text {; measuring range: } \\
500 \mathrm{~m} \cdot \mathrm{s}^{-2} \text {; frequency range: } 1 \sim 10000 \mathrm{~Hz}\end{array}$ & $\begin{array}{l}\text { Picking up acceleration } \\
\text { signal of plate vibration }\end{array}$ \\
\hline $\begin{array}{l}\text { Dynamic signal test and } \\
\text { analysis system }\end{array}$ & DH5922N & $\begin{array}{c}\text { Frequency range channel number: } 32 \text { channels; maximum power: } \\
100 \mathrm{~W} \text {; communication interface: USB3.0; power supply: } \\
220 \mathrm{VAC}, 12 \mathrm{VDC}\end{array}$ & $\begin{array}{l}\text { Collect force signal and } \\
\text { acceleration signal }\end{array}$ \\
\hline
\end{tabular}

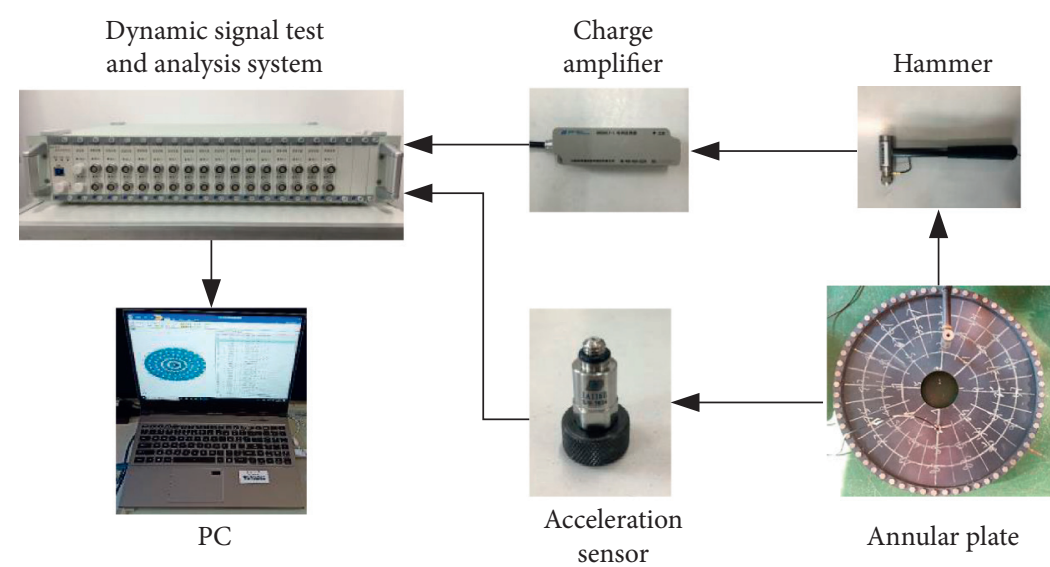

(a)

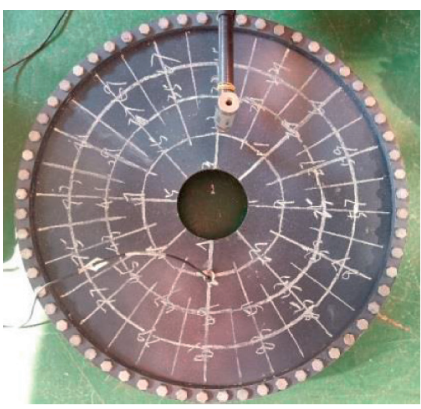

(b)

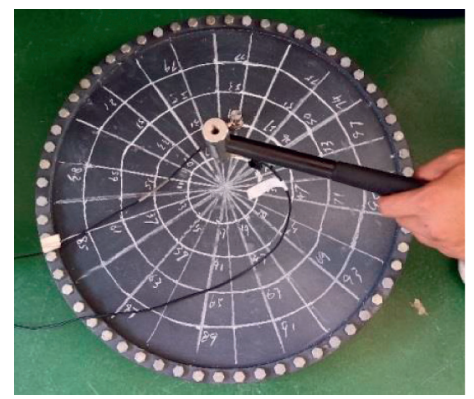

(c)

Figure 7: Schematic diagram of the model experiment and object of annular and circular plates. (a) Schematic diagram of the model experiment. (b) Annular plate with the F-C boundary condition. (c) Circular plate with the C boundary condition.

experiments of annular and circular plates are completed under the fixed boundary condition or the combination of free and fixed boundary conditions, which can provide more accurate experimental results. The experimental details on circular and annular plates can be found in $[38,39]$.

In this paper, the force measurement method is used for the model experiment. The measurement method is a singlepoint vibration pickup method, that is, the position of the acceleration sensor remains unchanged all the time, and the hammer strikes all measuring points in turn. This test method requires that the vibration pick-up point avoid the joint position of the test piece and tries to select the position with large deformation. During the experiment, the 32channel DH5922N dynamic signal test and analysis system and the corresponding modal analysis software are used, which were produced by Jiangsu Donghua Testing Technology Co., Ltd. In addition, the general piezoelectric acceleration sensor (1A116E) and the charge amplifier (DH5857-1) are also needed. Table 9 shows the experimental instruments and their related information used in the model experiment.

In this experiment, according to the experimental schematic diagram in Figure 7(a), the above experimental equipment and workpiece are installed and arranged. Figures 7(b) and 7(c) show the connection diagram of experimental equipment and test object of the annular plate and circular plate. In this experiment, the $30 \mathrm{~cm}$ thick foundation frame, $10 \mathrm{~cm}$ thick batten, and annular plate or circular plate are connected by 60 bolts with the same 
TABle 10: Specifications of test plates and arrangement.

\begin{tabular}{lcc}
\hline Name & Annular plate & Circular plate \\
\hline Internal radius of plate, $R_{0}(\mathrm{~m})$ & 0.05 & - \\
External radius of plate, $R_{1}(\mathrm{~m})$ & 0.25 & 0.25 \\
Plate thickness, $h(\mathrm{~mm})$ & 5 & 5.2 \\
Mass density, $\rho\left(\mathrm{kg} / \mathrm{m}^{3}\right)$ & 7850 & 7850 \\
Modulus of elasticity, $E(\mathrm{GPa})$ & 205.8 & 205.8 \\
Poisson's ratio, $\mu$ & 0.3 & 0.3 \\
Total number of measurement points & 88 & 97 \\
\hline
\end{tabular}
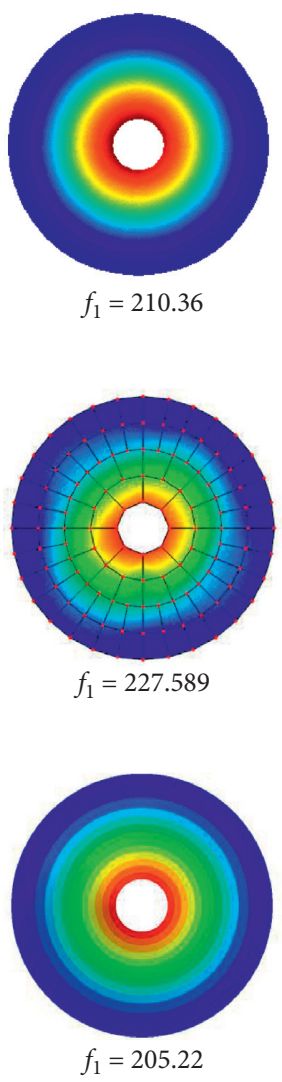
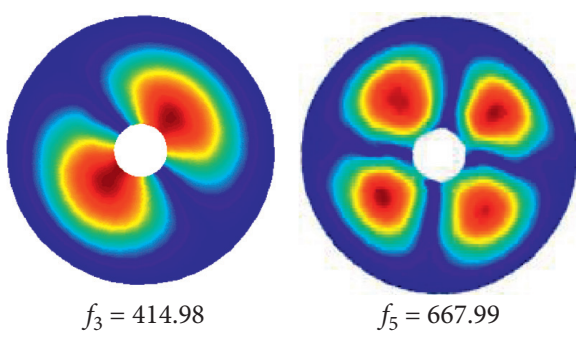

(a)
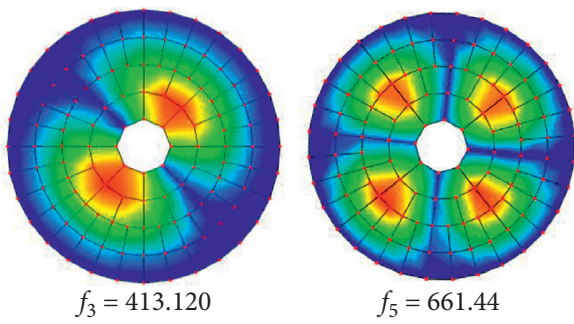

(b)
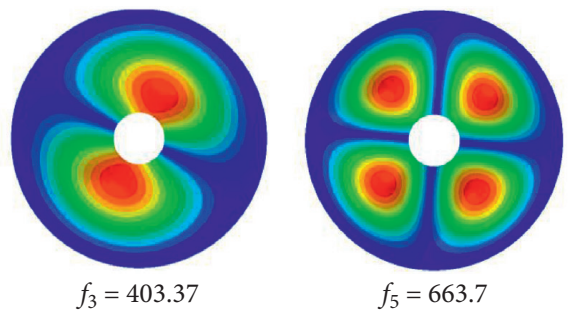
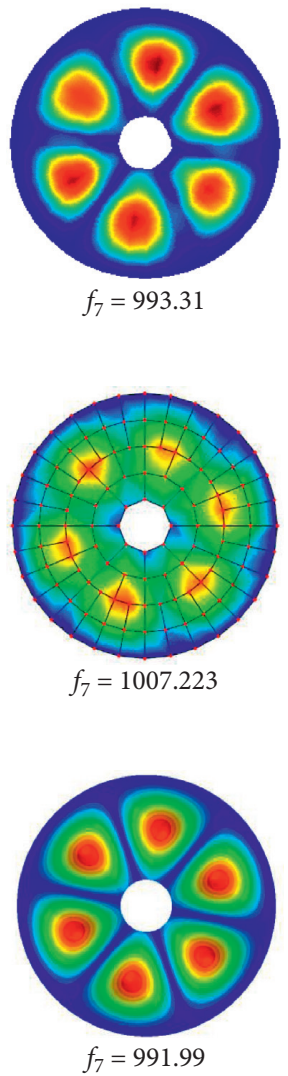

(c)

Figure 8: Comparison of experimental results for the annular plate with the F-C boundary condition. (a) Present results. (b) Experimental results. (c) FEM results.

diameter $8 \mathrm{~mm}$ to simulate the fixed boundary conditions. Table 10 gives specifications of test plates and arrangement. It should be noted that the geometric dimensions given in this table are all effective dimensions. The first test step is to select a point on the plate as the vibration pick-up point and place the acceleration sensor on it. In the second step, the hammer is used to excite each grid intersection on the plate, and the excitation force signal is measured by the force sensor on the hammer. Then, the acceleration sensor at the pick-up point of the plate collects the vibration acceleration signal and amplifies the signal through the charge adapter. Finally, using the force measurement method modal analysis software in the computer, modal parameters identification and frequency response function analysis are carried out on the experimental data. Through frequency response curve fitting, steady-state diagram calculation, and mode calculation, the vibration mode and natural frequency of the plate are finally obtained.

Figures 8 and 9 show the difference of experimental test results, FEM results, and present results. Among them, the maximum deviation of the experimental test results and present results is $7.57 \%$ for the natural frequency of the annular plate and $2.57 \%$ for the natural frequency of the circular plate. Besides, the maximum deviation of the experimental test results and FEM results is $9.83 \%$ for the natural frequency of the annular plate and $1.90 \%$ for the natural frequency of the circular plate. For the prediction results of the model shape, the two methods are highly consistent. The deviation of the above experimental results is within the acceptable range, which fully 

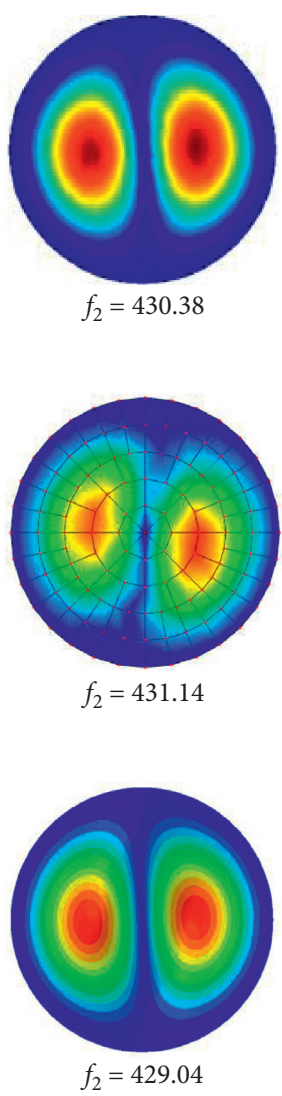
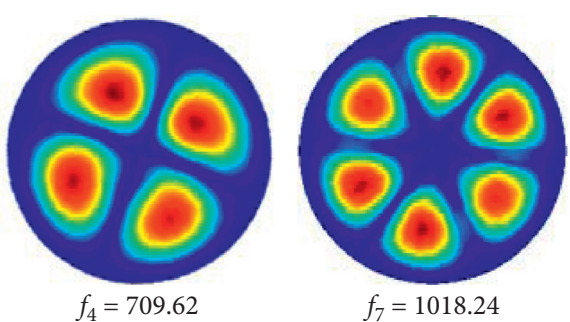

(a)
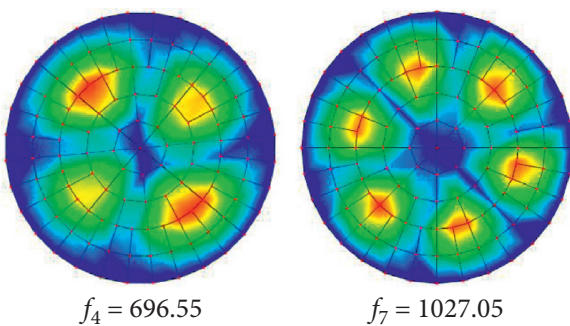

(b)
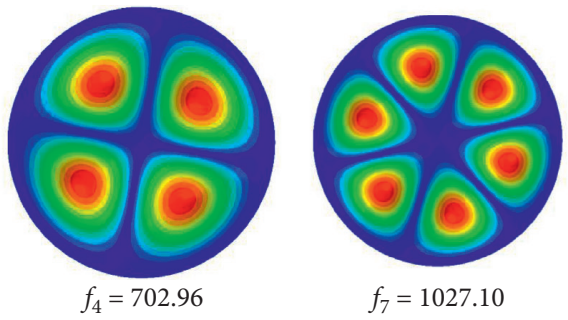
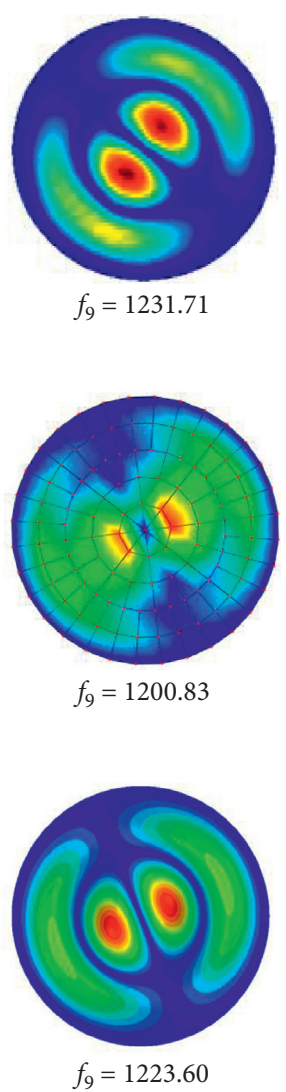

(c)

Figure 9: Comparison of experimental results for the circular plate with the fixed boundary condition. (a) Present results. (b) Experimental results. (c) FEM results.

proves the correctness of the analysis model for the vibration behaviors of annular and circular plates. The experimental error is caused by many reasons. First of all, the fixed boundary conditions of the plate cannot be fully simulated by the way of clamping the foundation frame and the battens. Then, the material parameters used in the numerical calculation of the plate deviate from the actual material parameters of the work piece, and the work piece cannot be completely ideal isotropic material. In addition, the accuracy deviation of the force sensor and acceleration sensor and the human error of the experimenter in the process of hammering will cause the error of experimental data.

\section{Conclusions}

This paper applies the Jacobi polynomial method to establish a unified analytical model for the vibration characteristics of laminated annular and circular plates under complex elastic boundary constraints based on Reddy's HSDT. Due to the adoption of multisegment segmentation technique, coupling springs are applied between adjacent segments to ensure continuity. In addition, various elastic boundary constraints are simulated by setting boundary springs at the edges of the plate. The Lagrangian energy equation of composite laminated annular or circular plates under elastic boundary constraints is established. The solution equation of free vibration behavior for the laminated plate is easily obtained by the Hamilton differential principle. On the basis of verifying the fast convergence and great accuracy of this method, further parametric study is carried out. Some important conclusions are revealed:

(1) Through the analysis of the stiffness value of the boundary springs, the value range of the elastic boundary condition is determined which are $10^{4}<k<10^{10}$ and $K<10^{4}, 10^{4}<k<10^{10}$ and $K>10^{10}$, $10^{4}<K<10^{10}$ and $k<10^{4}$, and $10^{4}<K<10^{10}$ and $k>10^{10}$. When $\theta=2 \pi$, the value of connective spring stiffness should be chosen as $10^{10}$ to ensure continuity.

(2) Material parameters, geometric parameters, and lamination schemes have an important effect on the vibration characteristics of the annular and circular plates. Specifically, the natural frequency will increase with the increase of plate thickness and anisotropy degrees.

(3) The frequency increases with the increase of the layer number and tends to be stable after the layer number exceeds 4 . Moreover, the frequency changes most significantly when the layer number changes from 1 to 2 . 
(4) The deviation of the model experimental results is within the acceptable range, which fully proves the correctness of the unified analysis model for the vibration behaviors of annular and circular plates.

\section{Data Availability}

The (TIF, OPTU, and DOCX) data used to support the findings of this study are available from the corresponding author upon request.

\section{Conflicts of Interest}

The authors declare that there are no conflicts of interest regarding the publication of this paper.

\section{Acknowledgments}

This project was supported by the National Key $R \& D$ Program of China (Grant no. 2018YFB2000100). The authors gratefully acknowledge the financial support from the National Natural Science Foundation of China (Grant no. 52005255), Natural Science Foundation of Jiangsu Province of China (Grant no. BK20200430), and Fundamental Research Funds for the Central Universities (Grant no. NS2020033).

\section{References}

[1] B. Afsharmanesh, A. Ghaheri, and F. Taheri-Behrooz, "Buckling and vibration of laminated composite circular plate on Winkler-type foundation," Steel and Composite Structures, vol. 17, no. 1, pp. 1-19, 2014.

[2] M. Amabili, R. Pierandrei, and G. Frosali, "Analysis of vibrating circular plates having non-uniform constraints using the modal properties of free-edge plates: application to bolted plates," Journal of Sound and Vibration, vol. 206, no. 1, pp. 23-38, 1997.

[3] E. Arshid and A. R. Khorshidvand, "Free vibration analysis of saturated porous FG circular plates integrated with piezoelectric actuators via differential quadrature method," ThinWalled Structures, vol. 125, pp. 220-233, 2018.

[4] A. Bahrami and A. Teimourian, "Free vibration analysis of composite, circular annular membranes using wave propagation approach," Applied Mathematical Modelling, vol. 39, no. 16, pp. 4781-4796, 2015.

[5] K. Mercan, A. K. Baltacioglu, and Ö. Civalek, "Free vibration of laminated and FGM/CNT composites annular thick plates with shear deformation by discrete singular convolution method," Composite Structures, vol. 186, pp. 139-153, 2018.

[6] K. K. Viswanathan, S. Javed, Z. A. Aziz, and K. Prabakar, "Free vibration of symmetric angle-ply laminated annular circular plate of variable thickness under shear deformation theory," Meccanica, vol. 50, no. 12, pp. 3013-3027, 2015.

[7] A. Powmya and M. C. Narasimhan, "Free vibration analysis of axisymmetric laminated composite circular and annular plates using Chebyshev collocation," International Journal of Advanced Structural Engineering, vol. 7, no. 2, pp. 129-141, 2015.

[8] K. Draiche, A. A. Bousahla, A. Tounsi, A. S. Alwabli, A. Tounsi, and S. Mahmoud, "Static analysis of laminated reinforced composite plates using a simple first-order shear deformation theory," Computers and Concrete, vol. 24, pp. 369-378, 2019.

[9] M. Amabili, P. Balasubramanian, R. Garziera, and G. RoyerCarfagni, "Blast loads and nonlinear vibrations of laminated glass plates in an enhanced shear deformation theory," Composite Structures, vol. 252, Article ID 112720, 2020.

[10] M. Amabili, "A new third-order shear deformation theory with non-linearities in shear for static and dynamic analysis of laminated doubly curved shells," Composite Structures, vol. 128, pp. 260-273, 2015.

[11] M. Amabili, Nonlinear Mechanics of Shells and Plates in Composite, Soft and Biological Materials, Cambridge University Press, Cambridge, UK, 2018.

[12] M. Amabili and J. N. Reddy, "The nonlinear, third-order thickness and shear deformation theory for statics and dynamics of laminated composite shells," Composite Structures, vol. 244, Article ID 112265, 2020.

[13] H. Bisadi, M. Es'haghi, H. Rokni, and M. Ilkhani, "Benchmark solution for transverse vibration of annular Reddy plates," International Journal of Mechanical Sciences, vol. 56, no. 1, pp. 35-49, 2012.

[14] E. Asadi and J. Jam, "Analytical and numerical buckling analysis of carbon nanotube reinforced annular composite plates," International Journal of Advanced Design and Manufacturing Technology, vol. 7, p. 35, 2014.

[15] M. Vinyas, A. Sandeep, T. Nguyen-Thoi, F. Ebrahimi, and D. Duc, "A finite element-based assessment of free vibration behaviour of circular and annular magneto-electro-elastic plates using higher order shear deformation theory," Journal of Intelligent Material Systems and Structures, vol. 30, no. 16, pp. 2478-2501, 2019.

[16] S. Hosseini-Hashemi, M. Es'haghi, and H. Rokni Damavandi Taher, "An exact analytical solution for freely vibrating piezoelectric coupled circular/annular thick plates using Reddy plate theory," Composite Structures, vol. 92, no. 6, pp. 1333-1351, 2010.

[17] S. Hosseini-Hashemi, M. Es'haghi, H. Rokni Damavandi Taher, and M. Fadaie, "Exact closed-form frequency equations for thick circular plates using a third-order shear deformation theory," Journal of Sound and Vibration, vol. 329, no. 16, pp. 3382-3396, 2010.

[18] M. M. Najafizadeh and H. R. Heydari, "An exact solution for buckling of functionally graded circular plates based on higher order shear deformation plate theory under uniform radial compression," International Journal of Mechanical Sciences, vol. 50, no. 3, pp. 603-612, 2008.

[19] M. Abualnour, A. Chikh, H. Hebali et al., "Thermomechanical analysis of antisymmetric laminated reinforced composite plates using a new four variable trigonometric refined plate theory," Computers and Concrete, vol. 24, pp. 489-498, 2019.

[20] O. Allam, K. Draiche, A. A. Bousahla et al., "A generalized 4unknown refined theory for bending and free vibration analysis of laminated composite and sandwich plates and shells," Computers and Concrete, vol. 26, pp. 185-201, 2020.

[21] N. Belbachir, M. Bourada, K. Draiche et al., "Thermal flexural analysis of anti-symmetric cross-ply laminated plates using a four variable refined theory," Smart Structures and Systems, vol. 25, pp. 409-422, 2020.

[22] N. Belbachir, K. Draich, A. A. Bousahla, M. Bourada, A. Tounsi, and M. Mohammadimehr, "Bending analysis of anti-symmetric cross-ply laminated plates under nonlinear thermal and mechanical loadings," Steel and Composite Structures, vol. 33, pp. 81-92, 2019. 
[23] M. Sahla, H. Saidi, K. Draiche, A. A. Bousahla, F. Bourada, and A. Tounsi, "Free vibration analysis of angle-ply laminated composite and soft core sandwich plates," Steel and Composite Structures, vol. 33, pp. 663-679, 2019.

[24] P. V. Katariya, S. K. Panda, and K. Mehar, "Theoretical modelling and experimental verification of modal responses of skewed laminated sandwich structure with epoxy-filled softcore," Engineering Structures, vol. 228, Article ID 111509, 2021.

[25] K. Mehar, S. K. Panda, and T. R. Mahapatra, "Theoretical and experimental investigation of vibration characteristic of carbon nanotube reinforced polymer composite structure," International Journal of Mechanical Sciences, vol. 133, pp. 319-329, 2017.

[26] J. N. Reddy, "A refined nonlinear theory of plates with transverse shear deformation," International Journal of Solids and Structures, vol. 20, no. 9-10, pp. 881-896, 1984.

[27] J. N. Reddy, "A simple higher-order theory for laminated composite plates," Journal of Applied Mechanics, vol. 51, no. 4, pp. 745-752, 1984.

[28] T. Ye, G. Jin, and Y. Zhang, "Vibrations of composite laminated doubly-curved shells of revolution with elastic restraints including shear deformation, rotary inertia and initial curvature," Composite Structures, vol. 133, pp. 202-225, 2015.

[29] C. Zhang, G. Jin, T. Ye, and Y. Zhang, "Harmonic response analysis of coupled plate structures using the dynamic stiffness method," Thin-Walled Structures, vol. 127, pp. 402-415, 2018.

[30] Z. Su, G. Jin, and T. Ye, "Electro-mechanical vibration characteristics of functionally graded piezoelectric plates with general boundary conditions," International Journal of $\mathrm{Me}$ chanical Sciences, vol. 138-139, pp. 42-53, 2018.

[31] Z. Su, G. Jin, and T. Ye, "Vibration analysis of multiplestepped functionally graded beams with general boundary conditions," Composite Structures, vol. 186, pp. 315-323, 2018.

[32] B. Qin, R. Zhong, Q. Wu, T. Wang, and Q. Wang, "A unified formulation for free vibration of laminated plate through Jacobi-Ritz method," Thin-Walled Structures, vol. 144, Article ID 106354, 2019.

[33] B. Qin, K. Choe, T. Wang, and Q. Wang, "A unified JacobiRitz formulation for vibration analysis of the stepped coupled structures of doubly-curved shell," Composite Structures, vol. 220, pp. 717-735, 2019.

[34] Q. Wang, K. Choe, J. Tang, C. Shuai, and A. Wang, "Vibration analyses of general thin and moderately thick laminated composite curved beams with variable curvatures and general boundary conditions," Mechanics of Advanced Materials and Structures, vol. 27, pp. 991-1005, 2019.

[35] Y. Kurylov and M. Amabili, "Polynomial versus trigonometric expansions for nonlinear vibrations of circular cylindrical shells with different boundary conditions," Journal of Sound and Vibration, vol. 329, no. 9, pp. 1435-1449, 2010.

[36] Y. Kurylov and M. Amabili, "Nonlinear vibrations of clamped-free circular cylindrical shells," Journal of sound and vibration, vol. 330, no. 22, pp. 5363-5381, 2011.

[37] G. Jin, T. Ye, X. Jia, and S. Gao, “A general Fourier solution for the vibration analysis of composite laminated structure elements of revolution with general elastic restraints," Composite Structures, vol. 109, pp. 150-168, 2014.

[38] E. Askari, K.-H. Jeong, and M. Amabili, "Hydroelastic vibration of circular plates immersed in a liquid-filled container with free surface," Journal of sound and vibration, vol. 332, no. 12, pp. 3064-3085, 2013.
[39] M. Kwak and M. Amabili, "Hydroelastic vibration of freeedge annular plates," Journal of Vibration and Acoustics, vol. 121, pp. 26-32, 1999. 